\title{
Effects of alga Fucus serratus decline on benthic assemblages and trophic linkages at its retreating southern range edge
}

\author{
Linney Duarte ${ }^{1, *}$, Francesca Rossi $^{2}{ }^{,}$Cristina Docal $^{3}$, Rosa M. Viejo ${ }^{1}$ \\ ${ }^{1}$ Área de Biodiversidad y Conservación, Universidad Rey Juan Carlos, 28933, Móstoles, Madrid, Spain \\ ${ }^{2}$ CNRS, UMR 9190 (MARBEC), Place Eugène Bataillon, Université de Montpellier, 34095 Montpellier, France \\ ${ }^{3}$ IMAR-CMA Instituto do Mar, Dep. Ciências da Vida, Universidade de Coimbra, 3004-517 Coimbra, Portugal
}

\begin{abstract}
Canopy-forming seaweeds are important coastal ecosystem engineers that sustain diverse multi-trophic assemblages. Their losses, with the subsequent reduction in habitat complexity, have been documented across many parts of the world and are often attributed to climate change and other anthropogenic factors. The general aim of the present study was to understand the repercussions of the decline of the canopy-forming alga Fucus serratus L. at its retreating southern range edge in the diversity and food-web linkages of intertidal assemblages. Few studies have attempted to document changes in benthic food webs following canopy loss. We examined the differences among southern locations situated at different distances from the range margin: those at the very edge, where $F$. serratus experienced a dramatic decline during recent years (marginal locations), and those where F. serratus is still dominant (central locations). Comparisons were made among locations situated at the same latitude and sharing a recent history of $F$. serratus dominance. Trophic relationships were analyzed using natural abundances of carbon and nitrogen stable isotopes. We report clear changes in the structure of benthic assemblages and lower trophic positioning of some consumers, suggesting an overall shrinkage of the food web length at the contracting range edge of $F$. serratus, which will transfer to higher trophic levels. Under present and future climatic scenarios, shifts in the distribution of coastal ecosystem engineers could entail a reorganization of local natural assemblages and food webs. More attention should be given to measure how much these shifts can modify the whole coastal food webs and their functioning.
\end{abstract}

KEY WORDS: Canopy-forming algae $\cdot$ Fucus serratus $\cdot$ Rear range edge margin $\cdot \delta^{15} \mathrm{~N} \cdot \delta^{13} \mathrm{C}$ Stable isotope analysis · Food web

Resale or republication not permitted without written consent of the publisher

\section{INTRODUCTION}

There is a global concern about the impacts of species loss in ecosystem functioning, which has generated a large body of research in recent years (see Hooper et al. 2005 for a review). Species loss is not a random process in nature, as some species are more susceptible than others to a variety of factors, including anthropogenic pressures and physical stress (Bracken et al. 2008). If the most sensitive species are those playing a key role in a natural system, their disappearance will alter the whole ecosystem functioning.

Canopy-forming seaweeds, such as fucoids and kelps, are important coastal ecosystem engineers that provide refuge for several species and sustain complex and diverse multi-trophic assemblages (Dayton 1985, Jones et al. 1997, Graham 2004, Schiel 2006). These seaweeds are an important part of the diet of basal macro-consumers at their adult stage or 
as algal sporelings, but also maintain a diverse community of algal epiphytes (Stephenson et al. 1986, Pavia et al. 1999, Van Alstyne et al. 1999). Their loss and the subsequent reduction in habitat complexity have been documented across many parts of the world and have often been attributed to climate change and other human-related stress factors (Airoldi et al. 2008, Byrnes et al. 2011, Wernberg et al. 2011, Strain et al. 2014). Hence, local extinctions of cool water canopy-forming algae have been documented at their contracting southern range margins, linked to recent ocean warming (e.g. Lima et al. 2007, Hawkins et al. 2009, Nicastro et al. 2013). A major challenge in ecology is not just to understand and predict changes in the distribution of key species under climate change, but also to identify the impacts of these changes on the associated assemblages and ecosystem functioning.

In temperate rocky shores, the loss of these canopyforming seaweeds frequently favors substrate colonization by small-sized turf-forming macroalgae (Strain et al. 2014 and references therein). The shift from canopy to turf-dominated substrates may change faunal species composition (Christie et al. 1998, Wikström \& Kautsky 2007) and reduce the resilience of the assemblages to large-scale disturbances (Eriksson et al. 2006, 2007). Despite indirect evidence suggesting that canopy loss has consequences that likely propagate along food chains (Airoldi et al. 2008), few studies have attempted to document these shifts in food-web structure. The scarce empirical analyses performed to date have detected reductions in foodweb complexity and length following canopy loss (Graham 2004, Byrnes et al. 2011).

A considerable number of canopy-forming, coldtemperate macroalgae, which dominate intertidal and shallow subtidal rocky shores of northern Europe, reach their southern range limit in the Iberian Peninsula (Lüning 1990). Among these species is the brown seaweed Fucus serratus L., which has 2 range boundaries in the NW Iberian Peninsula: one in northern Portugal, and another in northern Spain (Fischer-Piette 1957, Arrontes 1993). Recent and drastic declines in marginal populations of F. serratus have been reported in northern Spain, which are related to a rise in sea surface temperature and other emersion-linked physical variables (Viejo et al. 2011, Martínez et al. 2012a,b, Duarte et al. 2013).

The general aim of this paper was to understand the changes in the diversity and food-web linkages of intertidal benthic assemblages at the southern range limit of F. serratus in northern Spain, which is experiencing habitat shifts from the dominance of F. serra- tus to turf-forming algae. In particular, we examined whether there were differences in the benthic communities and trophic structure among southern locations situated at different distances from the range margin: those at the very edge, where F. serratus has experienced a dramatic decline during recent years (marginal locations), and those where F. serratus is still dominant (central locations). Comparisons were made among locations situated at the same latitude and sharing a recent history of $F$. serratus dominance. Due to the diverse array of food sources that canopies supply to consumers, we hypothesized that the drastic decline of this fucoid in marginal locations would affect the structure of the benthic food web, driving reductions in food-chain length and foodweb complexity.

Trophic relationships were analyzed using natural abundances of carbon and nitrogen stable isotopes (Peterson \& Fry 1987, Forero \& Hobson 2003). The $\delta^{15} \mathrm{~N}$ of animal tissue indicates trophic position, while the $\delta^{13} \mathrm{C}$ ratio can suggest diet sources (DeNiro \& Epstein 1978, Rau et al. 1983, Peterson \& Fry 1987). This technique has been largely used in studies on trophic structure and feeding habits in marine research (e.g. Rau et al. 1983, Forero \& Hobson 2003, Laurand \& Riera 2006). Nonetheless, relatively few stable isotope studies have been done in rocky shores (Bustamante \& Branch 1996, Bode et al. 2006, Hill \& McQuaid 2008, Riera et al. 2009), and much less frequent are studies evaluating the impact of loss of habitat-formers on the structure of coastal food webs (e.g. Byrnes et al. 2011, Coll et al. 2011).

\section{MATERIALS AND METHODS}

\section{Study locations}

The study was done in 4 locations on the northern coast of Spain distributed at different distances from the range margin of Fucus serratus. Two of the locations, Novellana $\left(43^{\circ} 34^{\prime} \mathrm{N}, 6^{\circ} 17^{\prime} \mathrm{W}\right)$ and Cadavedo $\left(43^{\circ} 33^{\prime} \mathrm{N}, 6^{\circ} 22^{\prime} \mathrm{W}\right)$ are situated in the marginal area, whereas San Pedro $\left(43^{\circ} 37^{\prime} \mathrm{N}, 7^{\circ} 19^{\prime} \mathrm{W}\right)$ and Area Longa $\left(43^{\circ} 36^{\prime} \mathrm{N}, 7^{\circ} 17^{\prime} \mathrm{W}\right)$ are about $100 \mathrm{~km}$ from the range boundary, hereafter called central area, but are in the same latitudinal position (Fig. 1). In each area, the 2 locations are exposed to moderate wave action and are about $10 \mathrm{~km}$ apart. In the central area, $F$. serratus is still abundant, whereas in the marginal area this species has experienced a drastic reduction in abundance during recent years (Arrontes 2002, Duarte et al. 2013). 


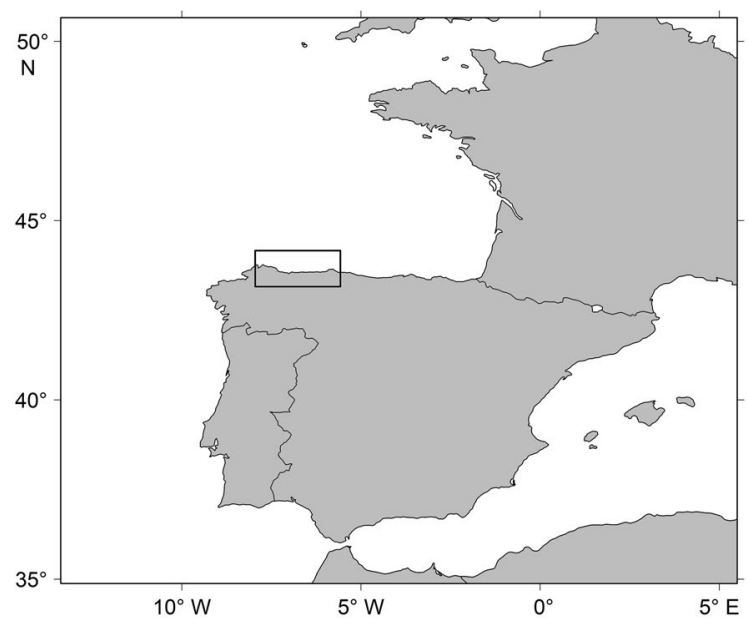

\section{Sample collection}

Field surveys were carried out in October 2007 and July 2008 during low spring tides at each location. These 2 sampling dates are representative of annual periods of low and high biomass of $F$. serratus in the study area (Arrontes 1993). Two sites per location separated by a distance of 50 to $100 \mathrm{~m}$ were randomly chosen at approximately $1.4 \mathrm{~m}$ above Lowest Astronomical Tide (maximum tidal range is around $4.3 \mathrm{~m}$ ). At each site and date, primary and secondary cover of macroalgae and sessile animals was estimated by randomly sampling $50 \times 50 \mathrm{~cm}$ plots $(\mathrm{n}=4)$. This was done with the point method using a grid with 81 regularly spaced points. Destructive sampling was then done by scraping the surface of plots of $30 \times 30 \mathrm{~cm}$, which were randomly selected at each site ( 3 and 2 samples per site in October and July, respectively) for abundance estimations of vagile fauna and for isotopic analyses of fauna and algae. All samples were stored frozen at $-20^{\circ} \mathrm{C}$ until sorted. They were then thawed and sieved at $1 \mathrm{~mm}$, and the animals retained were identified to the smallest possible taxonomic level, counted, and weighed after drying them at $60^{\circ} \mathrm{C}$ for $48 \mathrm{~h}$. Faunal species were identified with the help of distinct taxonomic keys (Chevreux \& Fage 1925, Lincoln 1979, Llera et al. 1983, González \& Méndez 1986). Abundant species (e.g. gastropods Bittium reticulatum and Rissoa spp. $>1000$ individuals in some samples) were sub-sampled to estimate total biomass per replicate.

\section{Isotopic analyses}

The most abundant macroalgae, including $F$. serratus, and most abundant invertebrates known to

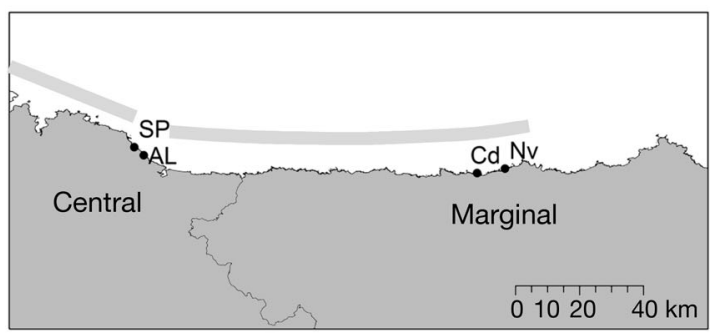

Fig. 1. Locations of study areas: San Pedro (SP) and Area Longa (AL) (central area), and Novellana (Nv) and Cadavedo (Cd) (marginal area). Grey line indicates the distributional range of Fucus serratus in northern Spain at the time of the study

consume benthic resources were analyzed for carbon and nitrogen isotopic composition. Reproductive tissue (receptacles) and vegetative parts of $F$. serratus were examined independently in July samples.

All samples (2-5 individuals per species and plot) were ground to a fine powder. The carbon and nitrogen isotopic composition of samples was determined using a Flash EA 1112 Elemental Analyzer coupled online via Conflo III interface to a Delta V Mass Spectrometer (Elemental Analyzer Isotope Ratio Mass Spectrometer). Acetanilide was used as a standard between samples. The carbon and nitrogen isotope ratios are expressed in delta $(\delta)$ notation, defined as the parts per thousand (\%) deviations from a standard material (urea and other elemental analyzer standards for $\delta^{13} \mathrm{C}$ and $\delta^{15} \mathrm{~N}$ ): $\delta^{13} \mathrm{C}$ or $\delta^{15} \mathrm{~N}=\left[\left(R_{\text {sample }} / R_{\text {standard }}\right)-1\right] \times 1000$, where $R={ }^{13} \mathrm{C} /{ }^{12} \mathrm{C}$ or ${ }^{15} \mathrm{~N} /{ }^{14} \mathrm{~N}$.

Precision in the overall preparation and analysis was better than $0.2 \%$ for both $\delta^{13} \mathrm{C}$ and $\delta^{15} \mathrm{~N}$. Animals $>5 \mathrm{~mm}$ were analyzed for stable isotopes after removal of their guts, shells, and any skeletal parts, while for small individuals $<5 \mathrm{~mm}$ (e.g. gastropods Rissoa spp., gammarid amphipods, and isopods) whole individuals were analyzed. Samples of small molluscs with shell and coralline algae were acidified to ensure removal of any carbonate residual. Acidification may introduce a bias in determining the natural abundance of ${ }^{15} \mathrm{~N}$ (Mateo et al. 2008). Even when the cuticle composition of crustaceans such as isopods includes calcite (Neues et al. 2007), their isotopic signal was similar with and without the acidification treatment and hence these samples were not acidified (ANOVAs for $\delta^{13} \mathrm{C}$ and $\delta^{15} \mathrm{~N}$ data for 5 species, $F_{1,8}=0.02, \mathrm{p}=0.90$ and $F_{1,8}=0.50, \mathrm{p}=0.49$, respectively). 


\section{Data analyses}

Permutational multivariate analyses of variance (PERMANOVA, Anderson et al. 2008) were used to test for differences among locations, sites, and dates in macroalgae and macrofauna assemblage compositions. Location and Date were considered fixed and orthogonal factors, and Site was treated as a random factor nested within Location. Abundance of fauna and percentage cover of algae were fourth-root transformed prior to the computation of triangular dissimilarity matrices based on the Bray-Curtis measures (Anderson et al. 2008). A posteriori pairwise comparisons were performed after significant pseudo- $F$ tests. Taxa contributing $>50 \%$ to the cumulative percentage of average dissimilarity were detected using SIMPER analyses (Clarke 1993).

Those faunal species represented at each location and date, and with a sufficient number of replicates, were analyzed to detect spatial and temporal changes in isotopic signatures by using 2-way ANOVAs, where Location and Date were treated as fixed and orthogonal factors. Also, 2-way ANOVA was employed to test differences between $F$. serratus reproductive and vegetative parts at central locations (July data). Prior to the ANOVAs, Cochran's C-test was used to check the homogeneity of variances (Underwood 1997). When significant differences for Location or its interaction with Date were found, Student-Newman-Keuls (SNK) tests were performed as a posteriori tests.

The inclusion of Site within Location as a factor in the ANOVAs of isotopic signatures was not feasible due to insufficient within-site replication for the target species at each Location. In order to test the variability among sites within each location, we plotted the average of $\delta^{13} \mathrm{C}$ and $\delta^{15} \mathrm{~N}$ values of each species found at one site vs. the values of the same species at the second site. Pearson's correlation coefficients (r) and the intercept and slope of Model II regressions (Major axis method, Legendre \& Legendre 1998) were then calculated. The level of association and relations close to 1:1 (i.e. intercepts non-significantly different from 0 and slopes not differing from 1) would indicate that species had similar average isotopic signatures between sites within each location.

PERMANOVAs and SIMPER analyses were performed using PRIMER v.6 (Anderson et al. 2008). ANOVAs were done using Statistica version 7.0. The Model II regressions were made with the R-package lmodel2 (version 1.7-0, R-Core Team 2012).

Dual-isotope plots were used to visually explore the relationships among benthic consumers and their potential food sources at each location and date. The relative trophic positions referred to selected isotopic baselines were estimated for the chitons Achantochitona spp. and the crab Pirimela denticulata. Since the chitons feed on macroalgae (e.g. Bode 1989), this group was considered the reference measure for the consumer. $P$. denticulata, on the other hand, is considered a predator/scavenger/omnivore (WoRMS Editorial Board 2014), but detailed information on its diet is unknown. Other intertidal crabs described as predators use a diversity of food items in their diets, including peracarid crustaceans (isopods, amphipods; Williams 1981, Cannicci et al. 2002). As these small crustaceans are dominated by grazing species, which display close and low $\delta^{15} \mathrm{~N}$ values (see 'Results'), they were used as the isotopic baseline for P. denticulata. The Bayesian mixing model SIAR 4.1 (Parnell et al. 2010; R-core Team 2012) was used to estimate the contribution of the potential food sources to the diet of the crab $P$. denticulata. The crab feeds on some of the collected food items only if its isotopic signal lies inside the area bounded by a convex polygon, as defined by the values of potential food sources corrected by selected fractionation values (Parnell et al. 2010 and references therein). These polygons were superimposed on the dualisotope plots to visually determine the importance of different food sources for the crab. For ${ }^{13} \mathrm{C}$ fractionation $\left(\Delta^{13} \mathrm{C}\right)$, we assumed a value of $0.3 \%$ o $( \pm 1.3 \mathrm{SD})$, as indicated by McCutchan et al. (2003) for consumers analyzed as a whole. For $\Delta^{15} \mathrm{~N}$, we selected the observed difference between the $\delta^{15} \mathrm{~N}$ of $P$. denticulata and the peracarid crustaceans in marginal locations, a mean value of $1.55 \%$ o $( \pm 0.38 \mathrm{SD})$. This value is similar to the average discrimination factor of $1.4 \%$ reported by Caut et al. (2009) for consumers raised on invertebrate diets; the value reported by Vanderklift $\&$ Ponsard (2003) for marine organisms (1.48\%o); and the range of $1-2 \%$ reported by Hill \& McQuaid (2008) for organisms in rocky shores of South Africa.

\section{RESULTS}

\section{Species composition and abundance of algae and faunal assemblages}

As expected, Fucus serratus cover was higher in the central locations of San Pedro and Area Longa than in the marginal locations of Cadavedo and Novellana, particularly if we consider secondary (canopy) cover (Fig. 2). Maximum average values of canopy cover were below $9 \%$ in marginal locations, while the mini- 


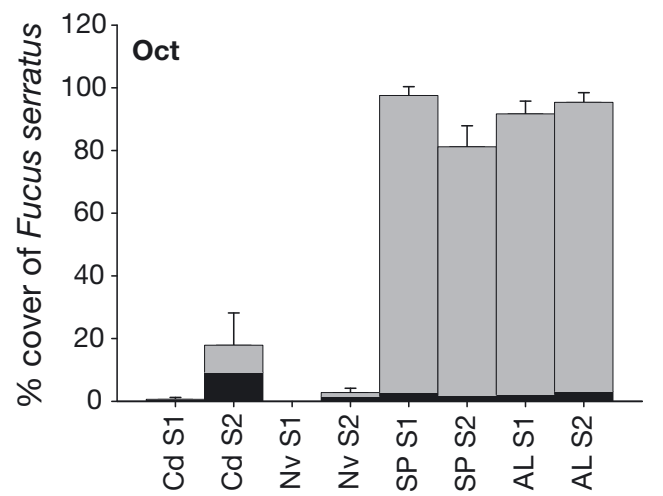

Marginal area Central area

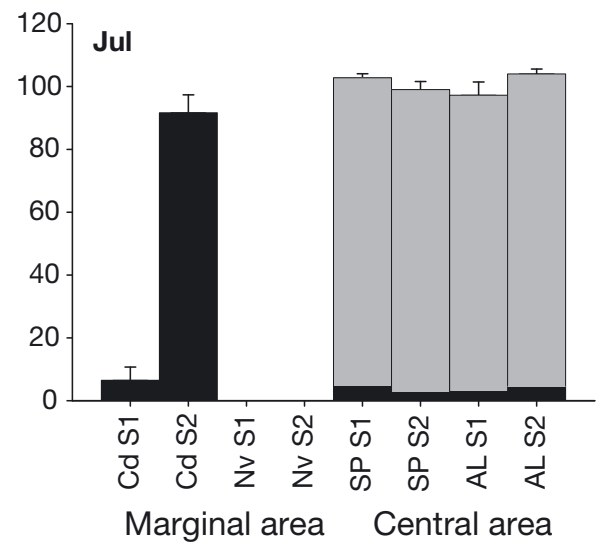

Fig. 2. Total cover (primary + secondary) of Fucus serratus in the studied sites (S1 and S2), locations, (abbreviations as in Fig. 1), and sampling dates (October 2007 and July 2008) (mean $\pm S E, n=4$ ). Black and grey bars indicate primary and secondary cover, respectively

mum average value in central locations was $>79 \%$ (see Fig. 2). Nevertheless, the primary cover of F. serratus greatly increased in July at one of the 2 marginal locations (Cadavedo; Fig. 2). Accordingly, the macroalgal assemblage showed differences between marginal and central locations in October, whereas in July Cadavedo did not differ from the central locations (PERMANOVA, Location $\times$ Date, $F_{3,48}=$ 2.17, $\mathrm{p}=0.049$; Cadavedo vs. central locations, $\mathrm{p}>$ 0.20 in July; for the complete analysis, see Table S1 in the Supplement at www.int-res.com/articles/suppl/ m527p087_supp.pdf). Also, small-spatial scale variability (sites within locations) was more constant over time in marginal than in central locations (PERMANOVA, Date $\times$ Site, $F_{4,48}=3.69, \mathrm{p}=0.001$; differences between sites within marginal locations, $\mathrm{p}<$ 0.05 for both dates; see Table S1 for the complete analyses). Apart from F. serratus, the macroalgae contributing to differences between marginal and central locations were opportunistic species from the genus Ulva and Ceramium, and the turf-forming alga Osmundea pinnatifida, which were more abundant in the marginal locations, whereas in central locations we observed a higher cover of erect and crustose coralline algae (SIMPER for October data; see Table S2 for the complete analysis). In July, these same species differentiated Novellana assemblages from those of Cadavedo and central locations, in addition to Leathesia difformis (more abundant in Novellana) and soft crustose species (more abundant in Cadavedo and central locations, SIMPER analysis, see Table S2).

Macrofaunal assemblages showed differences among the locations situated in the central and marginal areas of distribution of $F$. serratus. Overall, cen- tral locations were similar to each other, while marginal locations differed from each other (Table 1, a posteriori comparisons). The marginal location of Novellana differed from the other locations (Table 1, a posteriori comparisons), and these differences were in species abundances rather than in composition. On the other hand, Cadavedo presented an intermediate situation between Novellana and central locations. Novellana was characterized by higher densities of amphipods of the family Hyalidae, larval Diptera, and crustaceans (Tanaidacea), whereas the gastropods Rissoa spp., Barleeia unifasciata, B. reticulatum, and Ocenebra erinaceus, the chitons Acanthochitona spp., and the isopod Dynamene bidentata were more abundant in central locations and Cadavedo (Table 2). Dry weight data showed a similar pattern, as species like O. erinaceus, Rissoa spp., Gibbula spp., Hinia spp., Tricolia pullus, Patella spp., and Acanthochitona spp. differentiated central locations from the marginal location of Novellana (SIMPER analysis, see Table S3).

\section{Variation in the isotopic signatures of macrofauna among locations}

Four species were sufficiently represented across locations and dates to allow quantifying changes in their isotopic signatures (Table 3). The predator/ scavenger crab Pirimela denticulata and the grazer chitons Acanthochitona spp. showed clear differences between marginal and central locations in $\delta^{15} \mathrm{~N}$ values, which were on average 2.7 and $2.1 \%$, respectively, higher in central locations (Fig. 3a, Table 4). The other 2 taxa analyzed, the gastropods Bittium 
Table 1. Permutational ANOVA (PERMANOVA) for the effects of Date, Location, and Site on faunal species composition and abundance (as densities and dry weights) at mid-shore level, $\mathrm{n}=2$. A posteriori comparisons for differences between locations are also shown. The Monte Carlo asymptotic p-value was used both for the Location term in PERMANOVA and the pairwise comparisons. Location abbreviations as in Fig. 1

\begin{tabular}{|c|c|c|c|c|c|c|c|c|c|}
\hline \multirow[t]{2}{*}{ Source } & \multicolumn{4}{|c|}{$\longrightarrow$ Density } & \multicolumn{5}{|c|}{-Dry weight - } \\
\hline & $\mathrm{df}$ & MS & $F$ & \multicolumn{2}{|l|}{$\mathrm{p}$} & $\mathrm{df}$ & $\mathrm{MS}$ & $F$ & $\mathrm{p}$ \\
\hline Date, D & 1 & 3724.5 & 6.90 & $<0.001$ & & 1 & 3263.8 & 4.76 & 0.001 \\
\hline Location, L & 3 & 3719.9 & 6.44 & $<0.001$ & & 3 & 3456.0 & 4.36 & $<0.001$ \\
\hline Site (L) & 4 & 577.8 & 1.22 & 0.193 & & 4 & 793.6 & 1.74 & 0.010 \\
\hline $\mathrm{D} \times \mathrm{L}$ & 3 & 1357.9 & 2.51 & 0.005 & & 3 & 1225.7 & 1.79 & 0.059 \\
\hline $\mathrm{D} \times \mathrm{S}(\mathrm{L})$ & 4 & 540.3 & 1.14 & 0.289 & & 4 & 686.2 & 1.51 & 0.042 \\
\hline \multirow[t]{4}{*}{ Residual } & $22^{\mathrm{a}}$ & 473.3 & & & & 24 & 454.9 & & \\
\hline & & \multicolumn{4}{|c|}{$\begin{array}{r}\text { A posteriori comparisons } \mathrm{D} \times \mathrm{L} \\
\text { Density }\end{array}$} & & \multicolumn{3}{|c|}{$\begin{array}{c}\text { A posteriori comparisons } \mathrm{L} \\
\text { —Dry weight_- }\end{array}$} \\
\hline & & \multicolumn{2}{|c|}{ Oct } & \multicolumn{2}{|c|}{ Jul } & & & & \\
\hline & & $t$ & $\mathrm{p}$ & $t$ & $\mathrm{p}$ & & & & $\mathrm{p}$ \\
\hline \multirow[t]{2}{*}{ Within areas } & SP vs. AL & 1.60 & 0.090 & 1.32 & 0.194 & & & & 0.545 \\
\hline & Nv vs. Cd & 1.91 & 0.039 & 2.75 & 0.017 & & & & 0.007 \\
\hline \multirow[t]{4}{*}{ Between areas } & Nv vs. SP & 2.89 & 0.006 & 2.51 & 0.018 & & & & 0.008 \\
\hline & Nv vs. AL & 2.46 & 0.018 & 2.54 & 0.022 & & & & $<0.001$ \\
\hline & Cd vs. SP & 1.78 & 0.047 & 1.88 & 0.048 & & & & 0.152 \\
\hline & Cd vs. AL & 1.63 & 0.071 & 1.79 & 0.077 & & & & 0.017 \\
\hline
\end{tabular}

Table 2. Invertebrate species contributing to approximately the first $50 \%$ of the cumulative percentage of the average dissimilarities between Novellana ( $\mathrm{Nv}$ ) and the group of Cadavedo $(\mathrm{Cd})$ and central locations (SIMPER analysis of densities). The order of contribution of each species and the cumulative percentage of contribution are indicated. Location abbreviations as in Fig. $1, \mathrm{n}=4-6$ in Nv, $12-18$ in the group SP, AL, Cd (October to July, respectively)

\begin{tabular}{|c|c|c|c|c|c|c|c|c|}
\hline \multirow[t]{3}{*}{ Species } & \multicolumn{8}{|c|}{ Density (individuals per $90 \mathrm{~cm}^{2}$ ) } \\
\hline & \multirow{2}{*}{$\begin{array}{l}\text { SP, AL, Cd } \\
\text { Mean (SE) }\end{array}$} & \multirow{2}{*}{$\begin{array}{c}\text {-October- } \\
\text { Nv } \\
\text { Mean (SE) }\end{array}$} & \multicolumn{2}{|c|}{ Contribution } & \multirow{2}{*}{$\begin{array}{l}\text { SP, AL, Cd } \\
\text { Mean (SE) }\end{array}$} & \multirow{2}{*}{$\begin{array}{c}\text { Nv } \\
\text { Mean (SE) }\end{array}$} & \multicolumn{2}{|c|}{ Contribution } \\
\hline & & & Order & $\%$ & & & Order & $\%$ \\
\hline Rissoa spp. & $728.9(258.9)$ & $28.0(22.3)$ & 1 & 7.1 & $910.0(428.1)$ & $5.0(3.0)$ & 1 & 8.9 \\
\hline Hyalidae & $9.3(3.3)$ & $107.7(28.4)$ & 2 & 5.9 & $18.9(8.5)$ & $73.3(25.0)$ & 8 & 3.8 \\
\hline Tanaidacea & $4.1(1.8)$ & $73.0(25.8)$ & 3 & 5.2 & & & & \\
\hline Diptera (larvae) & $4.0(1.6)$ & $33.3(8.5)$ & 4 & 4.5 & $0.8(0.5)$ & $7.8(1.9)$ & 7 & 3.9 \\
\hline Barleeia unifasciata & $201.3(60.4)$ & $59.8(25.9)$ & 5 & 4.3 & $150.8(56.3)$ & $8.8(1.7)$ & 4 & 4.2 \\
\hline Bittium reticulatum & $831.3(223.4)$ & $420.3(98.8)$ & 6 & 4.0 & $914.7(212.2)$ & $463.8(161.3)$ & 6 & 3.9 \\
\hline Acanthochitona spp. & $35.7(5.8)$ & $3.7(1.9)$ & 7 & 3.7 & & & & \\
\hline Ocenebra erinaceus & $21.6(5.5)$ & $1.2(0.5)$ & 8 & 3.6 & $7.9(1.7)$ & $0.8(0.5)$ & 11 & 3.2 \\
\hline Skeneopsis planorbis & $5.4(3.5)$ & $25.2(22.6)$ & 9 & 3.4 & $32.4(15.1)$ & $8.8(5.1)$ & 5 & 4.0 \\
\hline Dynamene bidentata & $24.7(6.0)$ & $1.2(0.5)$ & 10 & 3.4 & $13.9(3.2)$ & $0.3(0.3)$ & 3 & 4.8 \\
\hline Microdeutopus spp. & $5.8(3.6)$ & $7.7(3.8)$ & 11 & 2.9 & $3.7(1.1)$ & $19.3(6.3)$ & 12 & 3.2 \\
\hline Nassarius spp. & $7.4(2.4)$ & $0.8(0.4)$ & 12 & 1.3 & $7.8(2.3)$ & $0.3(0.3)$ & 9 & 3.5 \\
\hline Tricolia pullus & & & & & $20.5(4.4)$ & & 2 & 5.6 \\
\hline Idotea spp. & & & & & $5.2(2.1)$ & & 10 & 3.4 \\
\hline Cumulative percentas & of dissimilarity & & & 47.4 & & & & 43.6 \\
\hline
\end{tabular}

reticulatum and Rissoa spp., presented $\delta^{15} \mathrm{~N}$ signatures 1.7 and $1.9 \%$, respectively, lower in the marginal location of Cadavedo (Fig. 3a, Table 4). Regarding the $\delta^{13} \mathrm{C}$ values, no clear pattern was evident between marginal and central locations. The $\delta^{13} \mathrm{C}$ values of Acanthochitona spp. and Rissoa spp. did not vary among locations, while those of $P$. denticulata and $B$. reticulatum were higher in the marginal location of Novellana than in the other locations (Fig. 3b, Table 4, SNK tests for P. denticulata: Novellana [Nv] > 
Table 3. List of taxa used for isotopic analyses. Locations and dates where samples were taken are shown (O: October 2007; J: July 2008; location abbreviations as in Fig. 1). Trophic groups (TG) are indicated; D: detritivore, G(ma/mi): grazer (macroalgal/microalgal), P: predator, Sc: scavenger, Pp: primary producer, Abb: abbreviations. Nomenclature follows WoRMS Editorial Board (2014) and Guiry \& Guiry (2014)

\begin{tabular}{|c|c|c|c|c|c|c|c|c|c|c|c|c|}
\hline \multirow[t]{2}{*}{ Species } & \multirow[t]{2}{*}{ Abb. } & \multirow[t]{2}{*}{ TG } & \multicolumn{4}{|c|}{ - Location- } & \multicolumn{2}{|c|}{$\delta^{13} \mathrm{C}$} & \multicolumn{2}{|c|}{$\delta^{15} \mathrm{~N}$} & \multirow[t]{2}{*}{$\mathrm{n}$} & \multirow[t]{2}{*}{ Source } \\
\hline & & & $\mathrm{Nv}$ & $\mathrm{Cd}$ & $\mathrm{AL}$ & $\mathrm{SP}$ & Mean & $\mathrm{SE}$ & Mean & SE & & \\
\hline \multicolumn{13}{|l|}{ Gastropoda } \\
\hline Rissoa spp. & $\mathrm{R}$ & $\mathrm{Gma} / \mathrm{mi}$ & $\mathrm{O}, \mathrm{J}$ & $\mathrm{O}, \mathrm{J}$ & $\mathrm{O}, \mathrm{J}$ & $\mathrm{O}, \mathrm{J}$ & -13.2 & 0.8 & 6.8 & 0.3 & 34 & Borja (1986) \\
\hline Gibbula spp. ${ }^{\mathrm{a}}$ & $\mathrm{G}$ & $\mathrm{Gma} / \mathrm{mi}$ & $\mathrm{O}$ & $\mathrm{O}, \mathrm{J}$ & $\mathrm{O}, \mathrm{J}$ & $\mathrm{O}, \mathrm{J}$ & -16.7 & 0.2 & 8.8 & 0.1 & 46 & Withers et al. (1975), Crothers (2001) \\
\hline Barleeia unifasciata & $\mathrm{Bu}$ & $\mathrm{D}, \mathrm{G}$ & $\mathrm{J}$ & $\mathrm{J}$ & J & $\mathrm{J}$ & -17.6 & 0.3 & 8.8 & 0.2 & 15 & Borja (1986) \\
\hline Bittium reticulatum & $\mathrm{Br}$ & $\mathrm{D}$ & $\mathrm{O}, \mathrm{J}$ & $\mathrm{O}, \mathrm{J}$ & $\mathrm{O}, \mathrm{J}$ & $\mathrm{O}, \mathrm{J}$ & -16.3 & 0.4 & 7.0 & 0.2 & 38 & Borja (1986) \\
\hline Nassarius spp. & $\mathrm{Na}$ & $\mathrm{Sc}$ & & J & $\mathrm{O}, \mathrm{J}$ & $\mathrm{O}, \mathrm{J}$ & -16.6 & 0.4 & 9.8 & 0.3 & 19 & Tallmark (1980) \\
\hline Tricolia pullus & $\mathrm{Tp}$ & $\mathrm{D}$ & $\mathrm{O}$ & $\mathrm{O}, \mathrm{J}$ & $\mathrm{O}, \mathrm{J}$ & $\mathrm{O}, \mathrm{J}$ & -13.4 & 1.0 & 8.3 & 0.3 & 18 & Fretter \& Manly (1977) \\
\hline Ocenebra erinaceus & Oc & $\mathrm{P}$ & & & J & $\mathrm{J}$ & -17.2 & 0.1 & 10.0 & 0.2 & 8 & Barry (1981) \\
\hline \multicolumn{13}{|l|}{ Polyplacophora } \\
\hline Acanthochitona spp. & $\mathrm{Ac}$ & $\mathrm{Gma} / \mathrm{mi}$ & $\mathrm{O}, \mathrm{J}$ & $\mathrm{O}, \mathrm{J}$ & $\mathrm{O}, \mathrm{J}$ & $\mathrm{O}, \mathrm{J}$ & -16.1 & 0.5 & 9.0 & 0.2 & 38 & Purchon (1977), Bode (1989) \\
\hline \multicolumn{13}{|l|}{ Isopoda } \\
\hline Dynamene bidentata & $\mathrm{Db}$ & Gma & $\mathrm{O}$ & $\mathrm{O}, \mathrm{J}$ & $\mathrm{O}, \mathrm{J}$ & $\mathrm{O}, \mathrm{J}$ & -14.1 & 0.3 & 6.8 & 0.3 & 34 & Holdich (1976), Arrontes (1990) \\
\hline Dynamene magnitorata & Dm & Gma & $\mathrm{O}$ & $\mathrm{O}, \mathrm{J}$ & $\mathrm{O}, \mathrm{J}$ & $\mathrm{O}, \mathrm{J}$ & -15.3 & 0.4 & 6.3 & 0.4 & 20 & Holdich (1976), Arrontes (1990) \\
\hline Idotea spp. & I & Gma & & & J & $\mathrm{J}$ & -16.5 & 0.2 & 7.9 & 0.3 & 4 & Salemaa (1987) \\
\hline Lekanesphaera sp. & $\mathrm{L}$ & $\mathrm{D}$ & & $\mathrm{J}$ & & & -15.5 & 0.6 & 5.2 & 0.1 & 4 & Mancinelli et al. (2005) \\
\hline \multicolumn{13}{|l|}{ Amphipoda } \\
\hline Hyalidae $^{\mathrm{d}}$ & $\mathrm{H}$ & Gma & $\mathrm{O}, \mathrm{J}$ & $\mathrm{O}, \mathrm{J}$ & $\mathrm{O}$ & $\mathrm{O}, \mathrm{J}$ & -18.9 & 0.3 & 7.0 & 0.2 & 25 & Viejo \& Arrontes (1992), Poore (1994) \\
\hline Ampithoe rubricata & $\mathrm{Ar}$ & Gma & $\mathrm{O}$ & $\mathrm{J}$ & $\mathrm{O}$ & $\mathrm{O}$ & -19.4 & 0.3 & 5.9 & 0.2 & 17 & Duffy \& Hay (1991) \\
\hline Ampithoe helleri & $\mathrm{Ah}$ & $\mathrm{D}, \mathrm{G}$ & & $\mathrm{J}$ & & & -21.5 & 1.2 & 4.9 & 0.0 & 2 & Guerra-García et al. (2014) \\
\hline Tanaidacea & $\mathrm{T}$ & $\mathrm{D}, \mathrm{G}$ & $\mathrm{O}, \mathrm{J}$ & $\mathrm{J}$ & & & -15.8 & 0.3 & 6.7 & 0.2 & 14 & $\begin{array}{l}\text { WoRMS Editorial Board (2014), Roca- } \\
\text { Torecilla \& Guerra-García (2012) }\end{array}$ \\
\hline \multicolumn{13}{|l|}{ Decapoda } \\
\hline Pilumnus hirtellus & $\mathrm{Ph}$ & $\mathrm{P}$ & & $\mathrm{O}$ & $\mathrm{O}$ & & -15.8 & 0.5 & 7.8 & 0.7 & 6 & Schaal et al. (2010) \\
\hline Pirimela denticulata & $\mathrm{Pd}$ & $\mathrm{P}, \mathrm{Sc}$ & $\mathrm{O}, \mathrm{J}$ & $\mathrm{O}, \mathrm{J}$ & $\mathrm{O}, \mathrm{J}$ & $\mathrm{O}, \mathrm{J}$ & -14.0 & 0.3 & 9.1 & 0.4 & 28 & WoRMS Editorial Board (2014) \\
\hline \multicolumn{13}{|l|}{ Polychaeta } \\
\hline Perinereis cultrifera & $\mathrm{PC}_{\mathrm{C}}$ & $\mathrm{P}$ & $\mathrm{O}$ & $\mathrm{O}$ & $\mathrm{O}$ & $\mathrm{O}$ & -18.8 & 0.3 & 8.6 & 0.3 & 14 & Schaal et al. (2010) \\
\hline \multicolumn{13}{|l|}{ Chlorophyta } \\
\hline Ulva rigida & Ur & $\mathrm{Pp}$ & $\mathrm{J}$ & $\mathrm{O}, \mathrm{J}$ & $\mathrm{J}$ & & -20.4 & 0.8 & 5.9 & 0.3 & 10 & \\
\hline Ulva clathrata & Uc & $\mathrm{Pp}$ & $\mathrm{O}$ & $\mathrm{O}$ & & $\mathrm{J}$ & -18.7 & 0.3 & 6.1 & 0.4 & 6 & \\
\hline \multicolumn{13}{|l|}{ Phaeophyta } \\
\hline \multicolumn{13}{|l|}{ Fucus serratus } \\
\hline (reproductive) & Frep & & & & J & J & -15.9 & 0.5 & 7.4 & 0.5 & 4 & \\
\hline Cladostephus spongiosus & $\mathrm{Cl}$ & $\mathrm{Pp}$ & $\mathrm{J}$ & $\mathrm{J}$ & & & -19.8 & 0.5 & 6.4 & 0.2 & 6 & \\
\hline Stypocaulon scoparium & $\mathrm{S}$ & $\mathrm{Pp}$ & $\mathrm{J}$ & $\mathrm{J}$ & & & -19.4 & 0.6 & 5.9 & 0.3 & 6 & \\
\hline \multicolumn{13}{|l|}{ Rhodophyta } \\
\hline Ceramium spp. & $\mathrm{Ce}$ & $\mathrm{Pp}$ & $\mathrm{O}, \mathrm{J}$ & $\mathrm{O}$ & & & -17.4 & 0.3 & 6.0 & 0.2 & 15 & \\
\hline Corallina spp. & Co & $\mathrm{Pp}$ & $\mathrm{O}, \mathrm{J}$ & $\mathrm{O}, \mathrm{J}$ & $\mathrm{O}, \mathrm{J}$ & $\mathrm{O}, \mathrm{J}$ & -13.1 & 0.7 & 5.8 & 0.2 & 37 & \\
\hline
\end{tabular}

San Pedro [SP] Cadavedo [Cd] > Area Longa [AL]; SNK test for $B$. reticulatum: $\mathrm{Nv}>\mathrm{SP} \sim \mathrm{AL} \sim \mathrm{Cd}$ ).

Differences among locations were not influenced by the sampling date (Table 4: non-significant interaction Date $\times$ Location). Six other consumers were well represented in October, and their isotopic values were tested for differences among locations (Fig. S1 in the Supplement). Three out of the 6 species varied in their $\delta^{15} \mathrm{~N}$ signature among locations, and only 1 showed clear-cut differences among the marginal and central locations. This species was the grazer isopod Dynamene bidentata, whose $\delta^{15} \mathrm{~N}$ signature was on average $1.7 \%$ o lower at marginal locations. The predator polychaete Perineireis cultrifera showed values $2 \%$ lower at the marginal location of Cadavedo, while the grazer gastropod T. pullus showed variability among locations, with lower values in marginal locations. Significant differences also occurred for $\delta^{13} \mathrm{C}$ values, but no clear trend between marginal vs. central locations was found (see Fig. S1). 
a

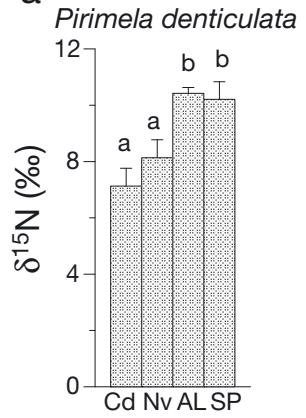

b

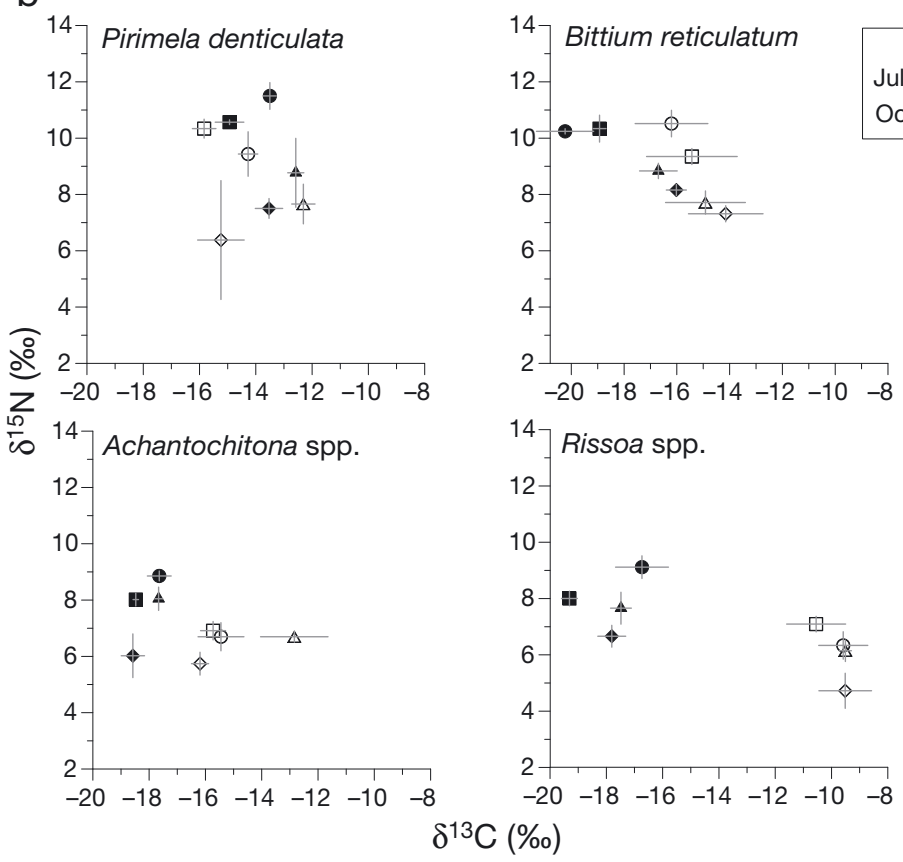

Fig. 3. (a) $\delta^{15} \mathrm{~N}$ values (mean $\left.\pm \mathrm{SE}, \mathrm{n}=6-10\right)$ for 4 consumers in marginal $(\mathrm{Cd}$, $\mathrm{Nv}$ ) and central (SP, AL) locations. Data from different sampling dates were pooled. Means sharing lowercase letters did not differ significantly based on SNK tests. (b) $\delta^{13} \mathrm{C}$ versus $\delta^{15} \mathrm{~N}$ signatures (mean $\pm \mathrm{SE}, \mathrm{n}=2-6$ ) for the same species. Location abbreviations as in Fig. 1

Variation in the isotopic signatures of food sources among locations

Isotopic signatures of the abundant macroalgae Corallina spp. and F. serratus did not show differences between central and marginal locations. Only the opportunistic algae Ulva spp. exhibited a trend of higher $\delta^{15} \mathrm{~N}$ values in central than in marginal locations (mean $\pm \mathrm{SE}=$ $7.3 \pm 0.3 \%, 5.9 \pm 0.1 \%$, $\mathrm{n}=4$ and 9 for central and marginal locations, respectively, July data). Corallina spp. showed differences between dates, but not among locations (ANOVA for the effect of Date in $\delta^{13} \mathrm{C}$ and $\delta^{15} \mathrm{~N}$ values, $F_{1,3}=63.80$, $\left.\mathrm{p}<0.001 ; F_{1,3}=13.96 \mathrm{p}=0.001\right)$. Thus, similarly to the consumer pattern, $\delta^{13} \mathrm{C}$ values were higher in October than in July (mean $\pm \mathrm{SE}=$ $-11.6 \pm 0.4 \%$ in October, $-15.1 \pm$ $1.2 \%$ in July, $\mathrm{n}=21$ and 16 , respectively), while the opposite trend was observed for $\delta^{15} \mathrm{~N}(4.8 \pm$ $0.2 \%$ in October, $7.0 \pm 0.2 \%$ in July). The $\delta^{15} \mathrm{~N}$ signature of the canopy-forming F. serratus differed among locations, with lowest values in Area Longa (ANOVA for the effect of Location, $F_{3,12}=4.50, \mathrm{p}=$ 0.025 , SNK tests, data from October, see Fig. 5).

The primary producers exhibited higher variability in carbon than in nitrogen isotopic composition, with Corallina spp. and reproductive

The small-scale, between-site variability of the ${ }^{15} \mathrm{~N}$ isotopic signature of species was relatively low within marginal and central locations in October (the between-site relationship did not differ from 1:1, high $\mathrm{r}$ values, Table 5), but it apparently increased at marginal locations in July (Fig. 4, Table 5, lower $\mathrm{r}$ values). In $\delta^{13} \mathrm{C}$ signatures, a trend of central vs. marginal was not detected (Table 5).

Regarding the temporal changes, overall $\delta^{13} \mathrm{C}$ values of the species were clearly higher in October than in July, particularly for Rissoa spp., whereas $\delta^{15} \mathrm{~N}$ values were higher in July than in October, although this trend was nonsignificant for $P$. denticulata (Fig. 3b, Table 4). parts of $F$. serratus (July data) presenting the highest $\delta^{13} \mathrm{C}$ values, and the vegetative parts of $F$. serratus and Ulva spp. the lowest (Figs. 5 \& 6).

The isotopic signatures of vegetative and reproductive parts (receptacles) of $F$. serratus were significantly different (ANOVA for $\delta^{13} \mathrm{C}$ and $\delta^{15} \mathrm{~N}$ values, $F_{1,8}=33.41, \mathrm{p}=0.001 ; F_{1,8}=13.77, \mathrm{p}=$ 0.006). Receptacles exhibited higher $\delta^{13} \mathrm{C}$ and $\delta^{15} \mathrm{~N}$ values than vegetative parts (Fig. $6, F$. serratus reproductive parts [Frep] vs. vegetative parts [Fs] in central locations; mean $\delta^{13} \mathrm{C}$ signature \pm $\mathrm{SE}=-15.9 \pm 0.5 \%$ for receptacles; $-22.1 \pm 0.7 \%$ for vegetative parts, and mean $\delta^{15} \mathrm{~N}$ signature \pm $\mathrm{SE}=7.4 \pm 0.3 \%$ and $5.5 \pm 0.3 \%, \mathrm{n}=4$ and 8 , 
respectively). Furthermore, this isotopic differentiation between reproductive and vegetative parts of $F$. serratus was greater than the variation detected among different algal species (Fig. 6, central locations).

Table 4. ANOVA for the effects of Date and Location in the $\delta^{13} \mathrm{C}$ and $\delta^{15} \mathrm{~N}$ isotopic signatures of 4 consumers; unbalanced data, $\mathrm{n}=2-6$. $\mathrm{D}=$ date;

$$
\mathrm{L}=\text { location }
$$

\begin{tabular}{|c|c|c|c|c|c|c|c|}
\hline \multirow{2}{*}{ Source } & \multirow{2}{*}{ df } & \multicolumn{3}{|c|}{$-\delta^{15} \mathrm{~N}-$} & \multicolumn{3}{|c|}{$-\delta^{13} \mathrm{C}$} \\
\hline & & MS & $F$ & $\mathrm{p}$ & MS & $F$ & $\mathrm{p}$ \\
\hline \multicolumn{8}{|c|}{ Pirimela denticulata $^{a}$} \\
\hline $\mathrm{D}$ & 1 & 8.524 & 4.315 & 0.050 & 4.038 & 5.841 & 0.025 \\
\hline $\mathrm{L}$ & 3 & 19.219 & 9.728 & $<0.001$ & 10.611 & 15.347 & $<0.001$ \\
\hline $\mathrm{D} \times \mathrm{L}$ & 3 & 1.054 & 0.534 & 0.664 & 1.017 & 1.470 & 0.251 \\
\hline Residual & 21 & 1.976 & & & 0.691 & & \\
\hline \multicolumn{8}{|c|}{ Bittium reticulatum $^{b}$} \\
\hline $\mathrm{D}$ & 1 & 13.823 & 17.082 & $<0.001$ & 85.097 & 34.716 & $<0.001$ \\
\hline $\mathrm{L}$ & 3 & 6.476 & 8.003 & $<0.001$ & 8.348 & 3.406 & 0.030 \\
\hline $\mathrm{D} \times \mathrm{L}$ & 3 & 1.380 & 1.705 & 0.187 & 3.490 & 1.424 & 0.255 \\
\hline Residual & 30 & 0.809 & & & 2.451 & & \\
\hline \multicolumn{8}{|c|}{ Rissoa spp. } \\
\hline $\mathrm{D}$ & 1 & 23.542 & 22.707 & $<0.001$ & 478.019 & 129.508 & $<0.001$ \\
\hline $\mathrm{L}$ & 3 & 7.197 & 6.941 & 0.001 & 5.550 & 1.504 & 0.237 \\
\hline $\mathrm{D} \times \mathrm{L}$ & 3 & 1.446 & 1.395 & 0.267 & 1.099 & 0.298 & 0.827 \\
\hline Residual & 26 & 1.037 & & & 3.691 & & \\
\hline \multicolumn{8}{|c|}{ Achantochitona spp. } \\
\hline $\mathrm{D}$ & 1 & 3.917 & 6.881 & 0.014 & 68.260 & 7.759 & 0.009 \\
\hline $\mathrm{L}$ & 3 & 14.473 & 25.427 & $<0.001$ & 18.318 & 2.082 & 0.124 \\
\hline $\mathrm{D} \times \mathrm{L}$ & 3 & 0.942 & 1.654 & 0.199 & 2.915 & 0.331 & 0.803 \\
\hline Residual & 29 & 0.569 & & & 8.797 & & \\
\hline \multicolumn{8}{|c|}{$\begin{array}{l}\text { an }{ }^{15} \mathrm{~N} \text { variances were homogeneous for a significance level of } 0.01 \\
\text { bIn } \delta^{13} \mathrm{C} \text { variances were heterogeneous, but the same results were } \\
\text { obtained with PERMANOVA }\end{array}$} \\
\hline
\end{tabular}

\section{Changes in trophic structure among locations}

The dual-isotope plots revealed complex trophic linkages among the analyzed benthic organisms (Figs. 5 \& 6). Primary consumers and macroalgae displayed similar $\delta^{13} \mathrm{C}$ ranges (Figs. $5 \& 6$ ). Among the crustacean grazers, the $\delta^{13} \mathrm{C}$ values of amphipods were close to the opportunistic and filamentous algae, but also to $F$. serratus, at least in October (Figs. 5 \& 6). Isopods also had $\delta^{13} \mathrm{C}$ signatures proximate to $F$. serratus, but mostly to reproductive parts (Frep in July, Fig. 6). The gastropods Rissoa spp., which exhibited the highest $\delta^{13} \mathrm{C}$ signatures among consumers in October, had values close to those of Corallina spp. at this date but not in July (Figs. 5 \& 6). Among secondary consumers, the $\delta^{13} \mathrm{C}$ signature of the polychaete $P$. cultriflera was close to the value of the amphipods and lower than the signal of the crab P. denticulata (see Fig. 5).

Primary consumers displayed high variability in their $\delta^{15} \mathrm{~N}$ signatures, with differences up to $4.5 \%$ (Figs. $5 \& 6$ ). Thus, the peracarid crustaceans (isopods, amphipods, and tanaids) presented overall $\delta^{15} \mathrm{~N}$ signatures similar to or even lower than the values of their potential macroalgal food sources (mean $\delta^{15} \mathrm{~N}=6.64 \%$, Figs. 5 \&6). The molluscs Gibbula spp., Achantochitona spp., T. pullus, and Barleeia unifasciata exhibited $\delta^{15} \mathrm{~N}$ values clearly higher than primary producers and similar

Table 5. Pearson's correlation coefficients (r) and parameters (intercept and slope) of Model II regressions (Major axis method) for the isotopic signatures of species between site 1 and 2 within each locality and sampling date. MA intcpt, MA slope = intercept and slope of the major axis regressions, respectively (correlation coefficients for $\delta^{15} \mathrm{~N}$ in Fig. 4). Whether the intercept differs from 0 and the slope differs from 1 (values included or not in the $95 \%$ confidence intervals) is indicated within brackets. $(\sim 0)$, $(\sim 1)$ : parameters did not differ from these values; $(<0)(<1)$ : parameters are lower than these values; ns: non-significant

\begin{tabular}{|c|c|c|c|c|c|c|c|c|}
\hline Area & Location & $\mathrm{r}$ & $\mathrm{p}$ & $\overline{\text { MA intcpt }}^{1}$ & ${ }^{3} \mathrm{C}-\overline{\text { MA slope }}$ & $\overline{\text { MA intcpt }}^{1}$ & ${ }^{5} \mathrm{~N}-$ & $\mathrm{n}$ \\
\hline \multicolumn{9}{|l|}{ Oct } \\
\hline Marginal & $\begin{array}{l}\text { Cadavedo } \\
\text { Novellana }\end{array}$ & $\begin{array}{l}0.722 \\
0.589\end{array}$ & $\begin{array}{l}0.028 \\
0.056\end{array}$ & $\begin{array}{c}-3.88(\sim 0) \\
\text { ns }\end{array}$ & $\begin{array}{c}0.68(\sim 1) \\
\text { ns }\end{array}$ & $\begin{array}{r}1.71(\sim 0) \\
-0.02(\sim 0)\end{array}$ & $\begin{array}{l}0.74(\sim 1) \\
1.07(\sim 1)\end{array}$ & $\begin{array}{r}9 \\
10\end{array}$ \\
\hline Central & $\begin{array}{l}\text { Area Longa } \\
\text { San Pedro }\end{array}$ & $\begin{array}{l}0.823 \\
0.948\end{array}$ & $\begin{array}{r}0.001 \\
<0.001\end{array}$ & $\begin{array}{r}-5.25(\sim 0) \\
1.76(\sim 0)\end{array}$ & $\begin{array}{l}0.69(\sim 1) \\
1.05(\sim 1)\end{array}$ & $\begin{array}{l}-0.01(\sim 0) \\
-2.21(\sim 0)\end{array}$ & $\begin{array}{l}1.03(\sim 1) \\
1.32(\sim 1)\end{array}$ & $\begin{array}{l}13 \\
14\end{array}$ \\
\hline Jul & & & & & & & & \\
\hline Marginal & $\begin{array}{l}\text { Cadavedo } \\
\text { Novellana }\end{array}$ & $\begin{array}{l}0.859 \\
0.812\end{array}$ & $\begin{array}{r}<0.001 \\
0.002\end{array}$ & $\begin{array}{l}0.10(\sim 0) \\
4.60(\sim 0)\end{array}$ & $\begin{array}{l}1.04(\sim 1) \\
1.21(\sim 1)\end{array}$ & $\begin{array}{c}0.84(\sim 0) \\
\mathrm{ns}\end{array}$ & $\begin{array}{c}0.85(\sim 1) \\
\text { ns }\end{array}$ & $\begin{array}{l}15 \\
10\end{array}$ \\
\hline Central & $\begin{array}{l}\text { Area Longa } \\
\text { San Pedro }\end{array}$ & $\begin{array}{l}0.971 \\
0.895\end{array}$ & $\begin{array}{l}<0.001 \\
<0.001\end{array}$ & $\begin{array}{l}-0.26(\sim 0) \\
-7.32(<0)\end{array}$ & $\begin{array}{l}0.97(\sim 1) \\
0.54(<1)\end{array}$ & $\begin{array}{l}-0.34(\sim 0) \\
-0.36(\sim 0)\end{array}$ & $\begin{array}{l}1.05(\sim 1) \\
1.03(\sim 1)\end{array}$ & $\begin{array}{l}13 \\
12\end{array}$ \\
\hline
\end{tabular}



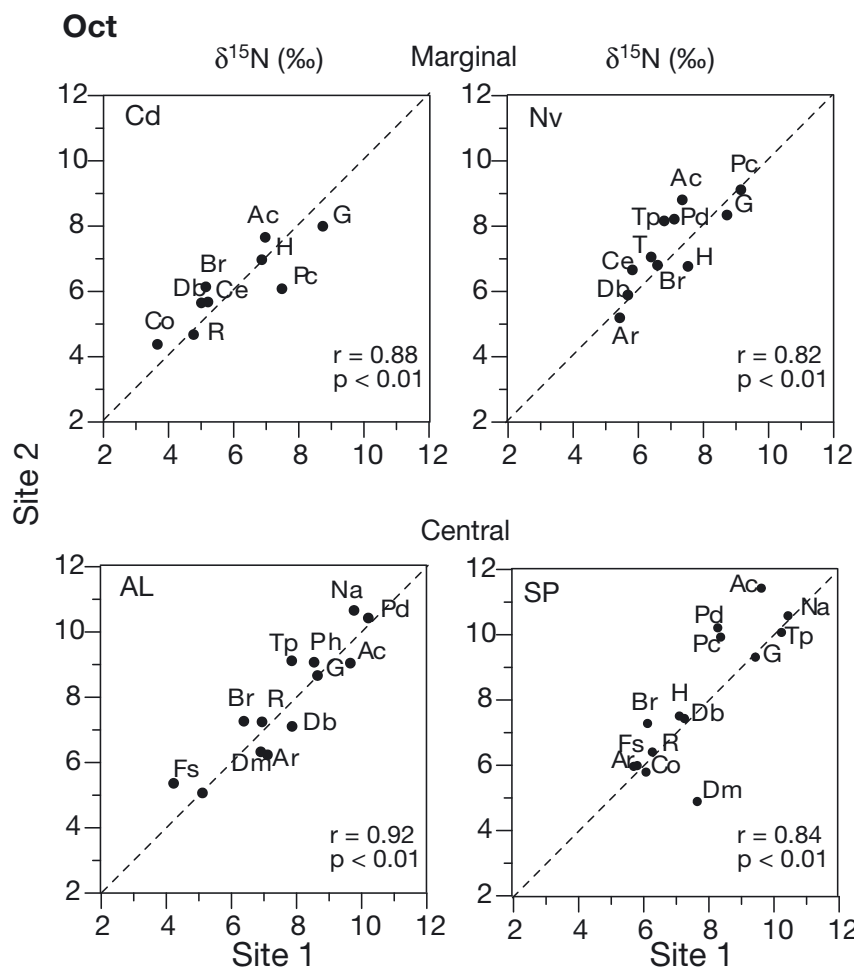

Central
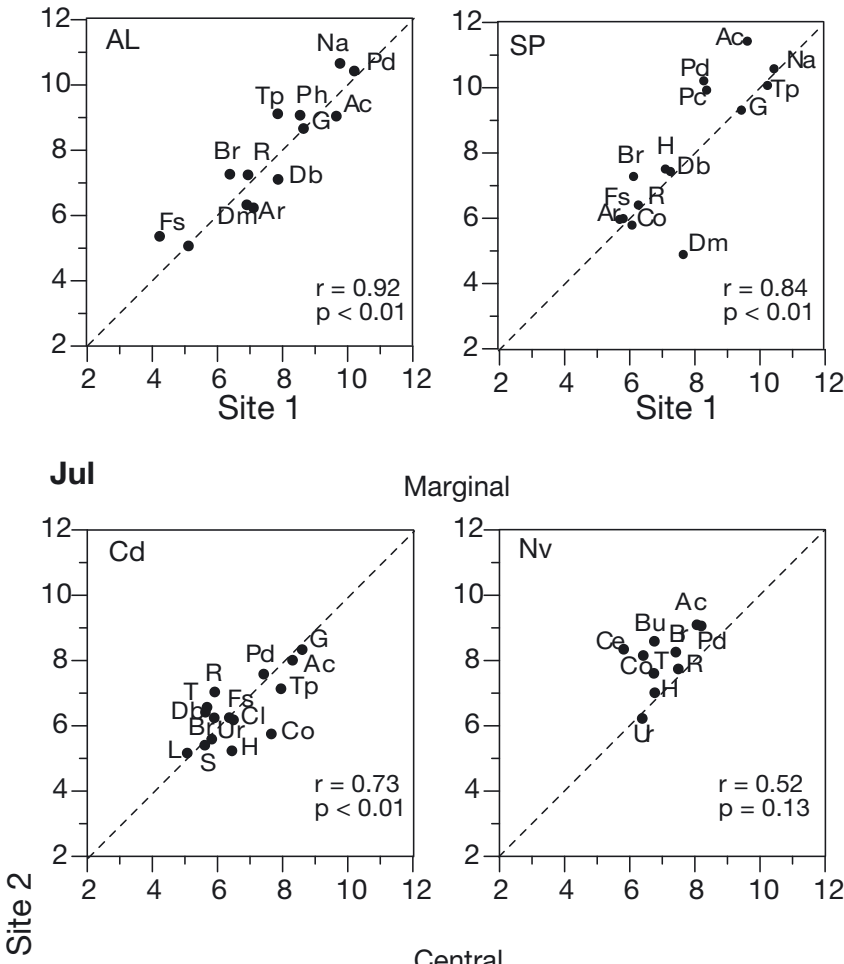

Marginal
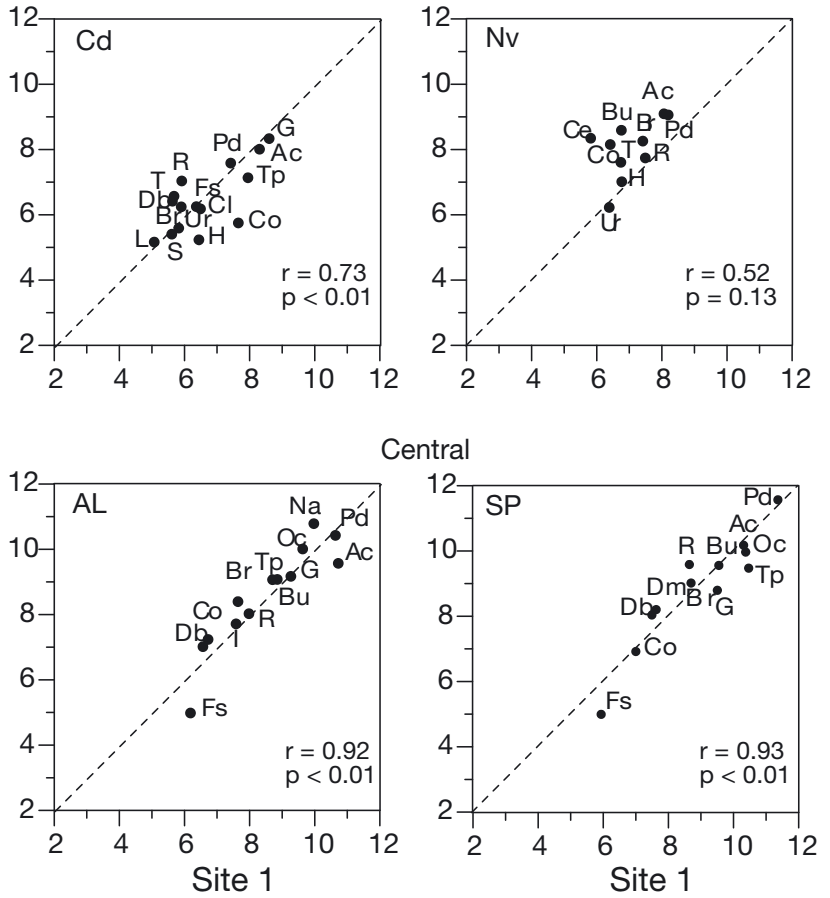

Fig. 4. Average $\delta^{15} \mathrm{~N}$ of species at Site 1 versus Site 2 within marginal and central locations at both sampling dates. Abbreviations of species and locations are indicated in Table 3 and in Fig. 1, respectively. Dashed line shows 1:1 correlation. Pearson's correlation coefficients (r) and $\mathrm{p}$-values are also indicated to or even higher than those of secondary consumers, predators, and scavengers (overall mean $\delta^{15} \mathrm{~N}=$ $8.78 \%$, Figs. $5 \& 6$ ). Finally, the $\delta^{15} \mathrm{~N}$ signatures of the small gastropods $B$. reticulatum and Rissoa spp. were similar to those of crustaceans in October, but closer to the other molluscs in July (Figs. 5 \& 6).

Interestingly, the dual-isotope plots also revealed apparent changes in the $\delta^{15} \mathrm{~N}$ signature and trophic position of some consumers between marginal and central locations and a general trend for benthic food webs to become shorter in marginal locations. Thus, the nitrogen range, i.e. the difference between the mean $\delta^{15} \mathrm{~N}$ signatures of species with the highest and the lowest values (see Figs. 5 \& 6), tended to be larger in central locations $(5.30 \pm$ $0.31 \%$ and $3.95 \pm 0.50 \%$ in central and marginal locations, respectively, mean $\pm \mathrm{SE}, \mathrm{n}=4$; data from different dates were pooled). In particular, the grazer chitons Achantochitona spp. and the predator/scavenger crab $P$. denticulata exhibited higher $\delta^{15} \mathrm{~N}$ values in central locations (see above). Also, their relative $\delta^{15} \mathrm{~N}$ signatures, i.e. the difference in relation to a baseline measure (see 'Materials and methods'), was clearly higher (around double) in central locations. For the chitons, the $\delta^{15} \mathrm{~N}$ values were $3.97 \pm 0.32 \%$ ond $2.10 \pm 0.05 \%$ in central and marginal locations, respectively (mean $\pm \mathrm{SE}$, $\mathrm{n}=4$; ANOVA $\left.F_{1,6}=33.54, \mathrm{p}=0.001\right)$ and $3.23 \pm 0.26 \%$ and $1.55 \pm 0.19 \%$ for $P$. denticulata $\left(F_{1,6}=22.87, \mathrm{p}=\right.$ 0.003). Rather than representing striking variations within species in the ${ }^{15} \mathrm{~N}$ discrimination factors $\left(\Delta^{15} \mathrm{~N}\right)$, these results feasibly reflect spatial changes in the diet and the trophic position of the consumers. Using the lowest value, $1.55 \%$, for average $\Delta^{15} \mathrm{~N}$ and $0.3 \%$ for $\Delta^{13} \mathrm{C}$ as fractionation factors, the diet of the crab P. denticulata appeared to be composed of macroalgae (Ceramium spp. and Corallina spp.), isopods (Dynamene spp.), and the small gastropods $B$. reticulatum and Rissoa spp. in marginal locations, whereas the consumer apparently excluded the algae and incorporated larger gastropods (Gibbula spp. and T. pullus) and the chitons Achantochitona spp. in central locations (see the convex polygon in Fig. 5, October data). The potential food sources varied from October to July, but again Gibbula spp. were included in the diet of crabs in central but not in marginal locations (Fig. 6). It was not possible to determine the range of proportions among these feasible food sources, as the variation among their isotopic signatures was limited and thus the Bayesian mixing model SIAR was subject to uncertainties (see Fig. S2 in the Supplement for results of SIAR with October data). 


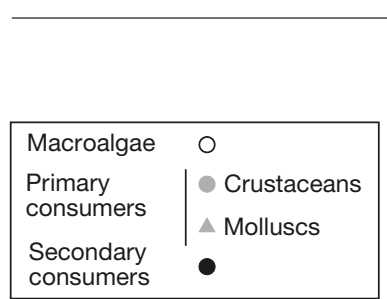

Fig. 5. $\delta^{13} \mathrm{C}$ versus $\delta^{15} \mathrm{~N}$ signatures (mean $\pm \mathrm{SE}, \mathrm{n}=2-6$ ) of organisms sampled in October in central and marginallocations. Vertical bars represent the $\delta^{15} \mathrm{~N}$ variation within the groups of secondary consumers (SC), primary consumers (PC), and macroalgae (M). The polygons represent the signatures of potential food sources corrected for fractionation (species indicated in gray font; Dynamene bidentata and $D$. magnitorata were pooled) for the crab Pirimela denticulata. Crosses indicate the positions of the corrected values of Gibbula spp. Location abbreviations as in Fig. 1. Abbreviations and diets of species are indicated in Table 3
Marginal
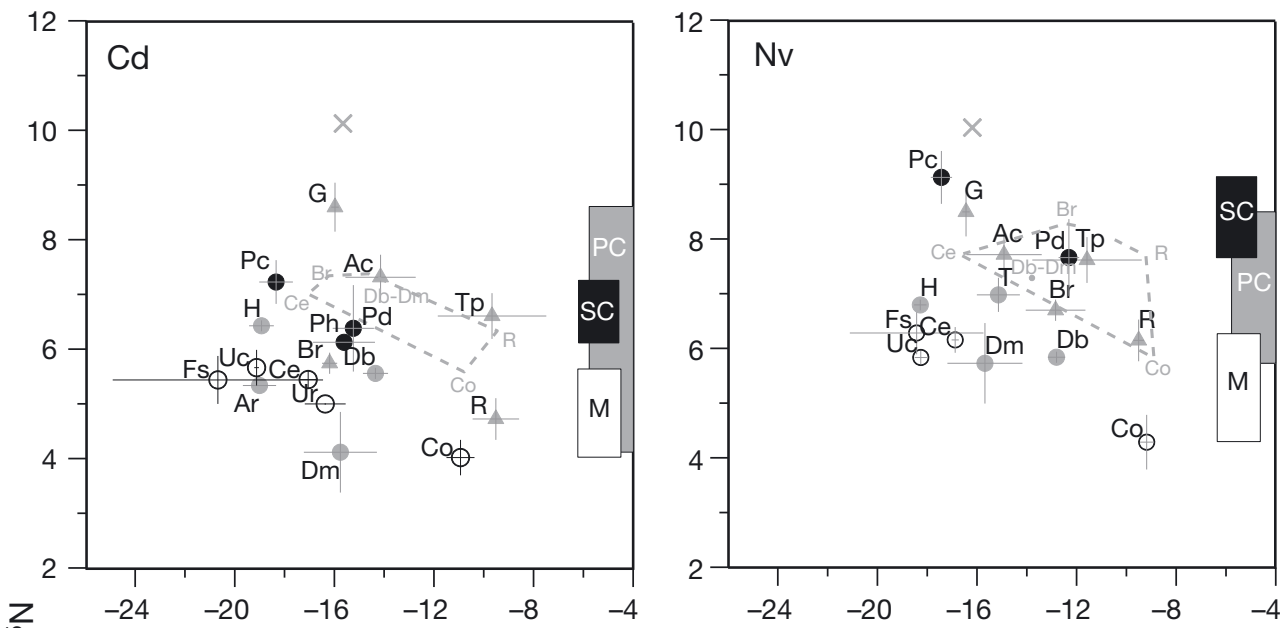

Central
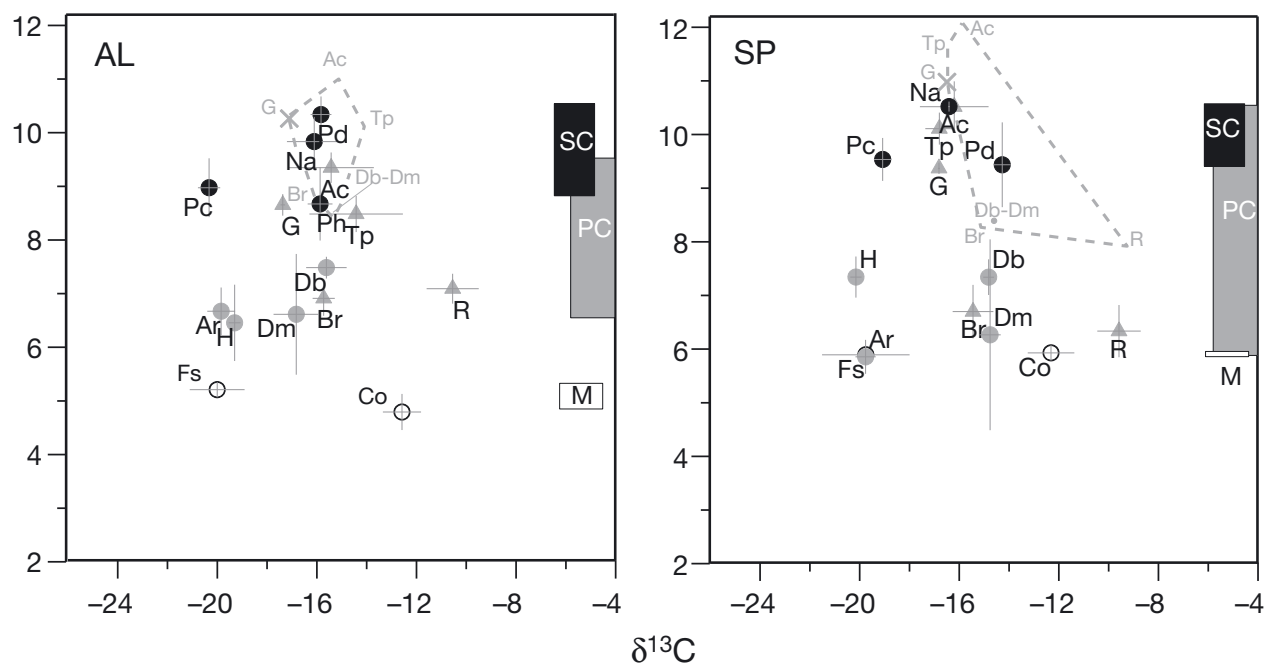

\section{DISCUSSION}

The loss of the canopy of Fucus serratus implies the transition from a multilayered macroalgal habitat (i.e. a canopy of the fucoid and species growing underneath) to a simpler habitat with a cover of smallersized algae. These habitat transformations, with growth enhancement of ephemeral and turf-forming species, have been previously reported in different geographical areas, as a consequence of abiotic stress or anthropogenic factors (Airoldi et al. 2008, Strain et al. 2014 and references therein). In our study, while erect and crustose coralline algae and soft crustose species proliferated under the canopy of $F$. serratus, in locations at the range edge, where the macroalgae were disappearing, opportunistic species (Ulva and Ceramium spp.) and the turf-forming Osmundea pinnantifida or the globular Leathesia difformis became more abundant. These might occur as a direct response to the canopy loss or, alternatively, both canopies and associated algae could be simultaneously reacting to environmental stressors. Canopies can control the development of the understory, limiting the growth of ephemeral, opportunistic algae and favoring other groups such as algal crusts or coralline species, feasibly by changing the abiotic conditions, i.e. reducing light, temperature, or attracting grazers (Lilley \& Schiel 2006, Eriksson et al. 2006, 2007).

Whereas the 'vertical' complexity of macroalgal assemblages was reduced due to the canopy loss, the horizontal small-scale variability (between-site variation) was higher within marginal locations (see 'Results'). This could reflect the patchy distribution of small-sized ephemerals and turf-forming species within boundary locations, but also the small-scale spatial pattern of $F$. serratus disappearance. Indeed, patches of $F$. serratus still remained at the end of this study in marginal locations (authors' pers. obs.). The remnants were dwarf morphs, short specimens with a bushy appearance, also detected in other Fucus 


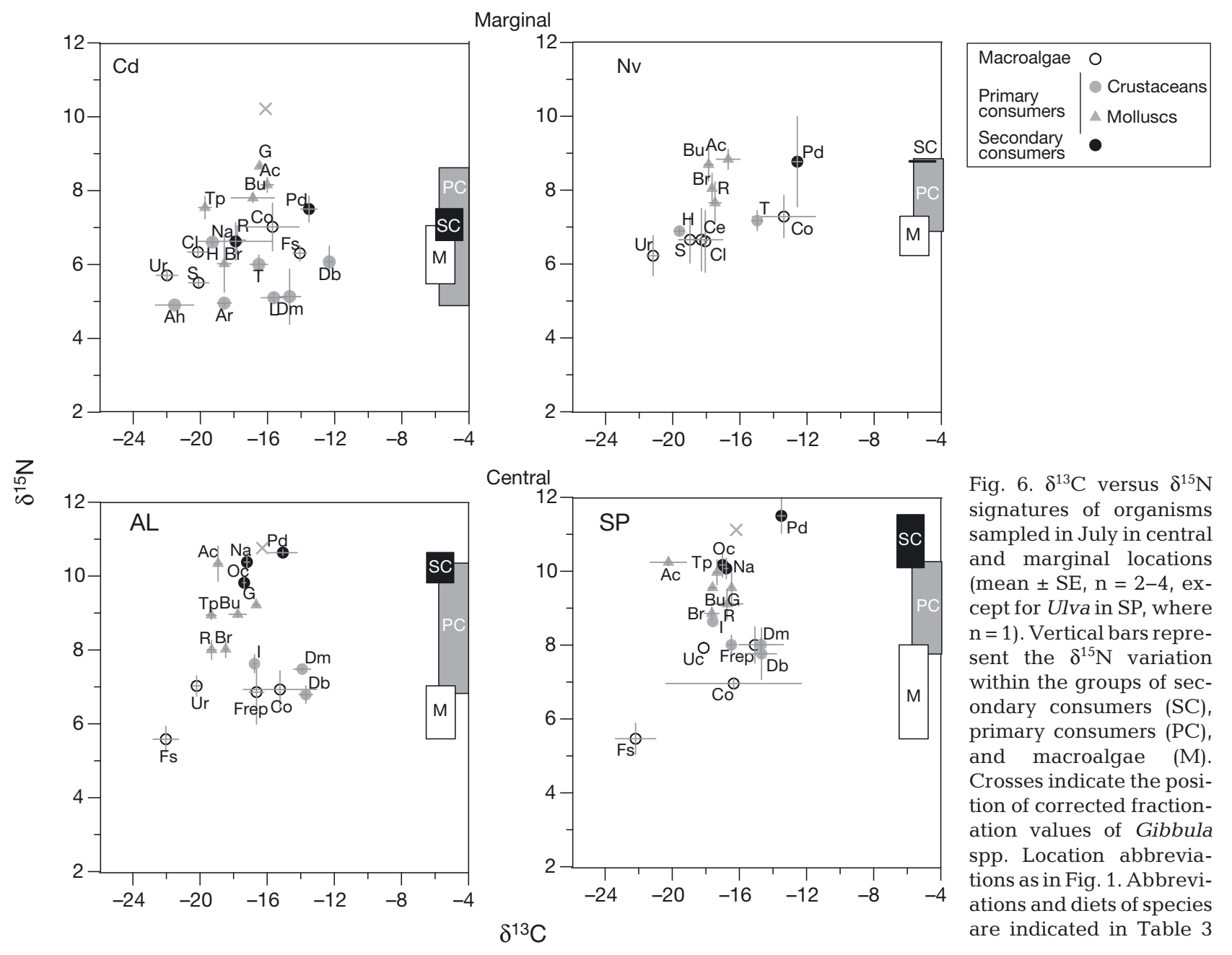

species (Viejo et al. 2011). The presence of remnants was more evident in Cadavedo than in Novellana, and this is why high values of primary cover were recorded at 1 site in Cadavedo, coinciding with the seasonal peak of F. serratus biomass (Arrontes 1993). A recent survey in 2013 showed that the seaweed became locally extinct in Novellana and other marginal locations, i.e. the range boundary moved westwards along a stretch of coast of about $80 \mathrm{~km}$ (towards the 'central' area), leaving behind the limit of a few sparse and very small populations, such as Cadavedo (R. M. Viejo pers. obs.; see also Duarte et al. 2013).

Macrofauna tracked the shifts in macroalgal communities, since invertebrate assemblages of Novellana differed from the remaining ones. As in previous studies that evaluated the influence of canopy loss on the associated fauna (e.g. Lilley \& Schiel 2006, Wikström \& Kautsky 2007), we observed reductions in abundance rather than in species composition. There was a shift in the dominant species in Novel- lana, where amphipods of the family Hyalidae, Tanaidacea, and larvae of Diptera were abundant, while gastropods, the chitons Acanthochitona spp., and the isopod Dynamene bidentanta were less numerous. The marginal location of Cadavedo, with the remnant presence of $F$. serratus, represented an intermediate situation with respect to changes in faunal assemblages. The effects of $F$. serratus disappearance on macrofauna may occur because canopy offers shelter from predators, changes hydrodynamics, and increases water retention at low tide, or because the lost seaweed constitutes an important food source (e.g. Arrontes 1999 and references therein, Bates \& DeWreede 2007, Christie et al. 2009, Best et al. 2014). Canopies provide a diverse array of food sources for consumers, including the seaweed itself, which could be directly eaten by grazers or as detritus (Fredriksen 2003, Norderhaug et al. 2003, Schaal et al. 2010), but also support the micro- and macroalgal epiphytes, sessile animals, and microorganisms growing on the thallus surface (Pavia et al. 1999, Lachnit et al. 2013). 
The preferences for food and habitat vary among faunal species. The isopod $D$. bidentata, which was more abundant in central locations, has been commonly associated with canopy-forming algal hosts upon which the isopod feeds preferentially (Arrontes 1990, Viejo \& Arrontes 1992, Morán \& Arrontes 1994). On the other hand, the higher abundance of hyalid amphipods and dipteran larvae in the marginal location of Novellana might be related to their food preferences, as they feed on different resources, including filamentous and ephemeral species such as Ulva spp. (Henriques-Oliveira et al. 2003, Taylor \& Steinberg 2005, Guerra-García et al. 2014). Our stable isotope analyses (the $\delta^{13} \mathrm{C}$ signatures) indicated that the isopod $D$. bidentata could be feeding on $F$. serratus, in particular on reproductive parts, while amphipods may also consume species of Ulva or Ceramium. Moreover, turf-forming and filamentous algae provide better shelter from predation and a more adhesive surface for amphipods than erect flat fronds (Buschmann 1990, Hacker \& Steneck 1990), such as those of F. serratus.

Benthic food webs, supported by macrophytes (macroalgae or sea grasses), are generally short in length when they are compared to other ecosystems, since most invertebrates are detritivores or herbivores and have high levels of omnivory (Fredriksen 2003, Hill \& McQuaid 2008, Baeta et al. 2009, Schaal et al. 2010). Nonetheless, the presence of habitatforming macrophytes by increasing habitat complexity may raise diversity within and among trophic levels, leading omnivore species to feed at higher trophic levels, thereby enhancing food-web complexity (Coll et al. 2011, Rossi et al. 2015). Overall, low nitrogen ranges $(\sim 4-5 \%$ o) were detected in the studied rocky-shore systems, revealing the short length of the food webs. Moreover, the observed discrepancies between the discrete trophic groups in which the species were a priori classified, and their $\delta^{15} \mathrm{~N}$ signatures, indicated the presence of intra-guild variability in the diets and high levels of omnivory. In particular, grazing crustaceans (isopods, gammarid amphipods) presented $\delta^{15} \mathrm{~N}$ signatures similar to or even lower than the values of their potential macroalgal food sources, while grazing gastropods such as Gibbula spp. and the chitons Achantochitona spp. exhibited $\delta^{15} \mathrm{~N}$ values $\sim 2 \%$ higher on average, proximate to the isotopic signal of secondary consumers. Differences in $\delta^{15} \mathrm{~N}$ signatures among primary consumers were up to $4.5 \%$ o (see Figs. $5 \& 6$ ). Low fractionation levels of small crustacean grazers (e.g. McCutchan et al. 2003, Michel 2011) and higher ${ }^{15} \mathrm{~N}$ enrichment of chitons and the topshells Gibbula spp. were reported in previous studies (Fredriksen 2003, Riera et al. 2009, Schaal et al. 2010). A high variability within the group of primary consumers might reflect differences between species in trophic fractionation, due to metabolic and assimilation processes, such as the form of $\mathrm{N}$ excretion (Vander Zanden \& Rasmussen 2001, Vanderklift \& Ponsard 2003). Both crustacean and marine molluscs are, however, primarily ammonotelic. Moreover, the observed range of $\delta^{15} \mathrm{~N}$ variation within the group of primary consumers is probably too large to be explained only by these differences and rather reflects the variability in their feeding modes and diets. Chiton and gastropod species use their radulas to scrape a diversity of food sources on hard substrata, and in the case of the topshell, on the surface of the thalli of macroalgae (Bode 1989, Crothers 2001, Bhatnagar \& Bansal 2009, Schaal et al. 2010). Their high $\delta^{15} \mathrm{~N}$ signatures, close to predators and scavengers ( $>10 \%$ for Achantochitona spp. at some locations, see Figs. 5 \& $6)$, indicated that they may also include animals in their diets. This is in accordance with the results of Camus et al. (2008), who found that the ingestion of animals was a frequent phenomenon in molluscan 'herbivores' of Chilean coasts, and consequently these species presented a high potential for omnivory. Even when molluscs may feed on remains of vagile animals and sessile fauna, in our study the tidal zone dominated by macroalgae presented low coverage of sessile animals such as barnacles (average percentage covers $<0.7 \%$ ), without apparent differences between central and marginal locations.

Remarkably, our stable isotope results also revealed food-web disruptions at the range boundary of F. serratus, with shifts in the diet and lower trophic positioning of some consumers, and an overall trend for shrinkage of the food-web length at marginal locations. In particular, the $\delta^{15} \mathrm{~N}$ signatures of the chitons Achantochitona spp. and the crab Pirimela denticulata fell in marginal locations (both the absolute values but also the relative values with respect to their respective isotopic baselines, i.e. macroalgae and grazer peracarid crustaceans). The estimation of the trophic positioning of organisms is moderately sensitive to the selection of the trophic baseline (Post 2002). However, the spatial shift in the $\delta^{15} \mathrm{~N}$ signatures of the crab persisted with respect to macroalgae as a food source (average differences in central vs. marginal locations: 4.3 and $1.7 \%$, respectively), or even with respect to Ulva spp. (3.6 vs. $2.2 \%$ ), the only primary producer showing spatial trends. Furthermore, trophic positioning is very sensitive to the considered value of trophic fractionation of $\delta^{15} \mathrm{~N}$ (Post 
2002), and this discrimination factor is variable, which also adds difficulties to the reconstruction of diets in natural systems (Post 2002, Caut et al. 2009). Nevertheless, we interpret these consistent differences in the average $\delta^{15} \mathrm{~N}$ signal of the 2 consumers between marginal and central locations as shifts in their diets and evident changes in their trophic positioning. We based this interpretation on the fact that differences were sustained with respect to trophic baselines, and they were similar to or greater than the discrimination factors reported by different authors (see 'Materials and methods'). Our results then suggest that consumers, such as the chitons Achantochitona spp. and the crab P. denticulata, increased the proportion of animals in their diets at central locations, i.e. in the presence of canopies. In particular, the crab might consume macroalgae and small gastropods at marginal locations, while excluding the algae and incorporating larger gastropods (Gibbula spp.) and even the chitons Achantochitona spp. at central locations. These diet shifts are in accordance with the diverse diet of intertidal crabs, with species considered to be predators very often ingesting macroalgae (Williams 1981, Cannicci et al. 2002). Moreover, the changes are in agreement with the observed shifts in faunal assemblages, as large molluscs, such as Gibbula spp., and Acanthochitona spp. were more abundant in the presence of $F$. serratus. We applied average fractionation values which were close to those reported in the literature by different authors in comparable systems (see 'Materials and methods'). Furthermore, the use of larger ${ }^{15} \mathrm{~N}$ discrimination factors from the literature (e.g. $2.5 \pm$ $2.5 \%$, Vander Zanden \& Rasmussen 2001) would not allow for estimations of the diet of the crab at marginal locations, as the corrected values of the potential food items would lie well above the signal of the consumer. The use of fixed average fractionation values, in particular the measured $\Delta^{15} \mathrm{~N}$ of $1.55 \%$, requires cautious interpretation of the detailed, finegrained variations in the crab diet. Yet, our results indicate that consumers feasibly fed more as secondary consumers in the presence of canopies and increased their consumption of algae when the species that formed the canopy was lost.

Greater between-site variability in the $\delta^{15} \mathrm{~N}$ signatures was also detected in July at marginal locations. This could be related to the mosaic of patches of ephemerals, turf-forming species, and remnants of $F$. serratus, which might provoke diet shifts of consumers at these fine-grained scales in these locations (see also Schaal et al. 2011). Alternatively, it could be the result of small spatial shifts in the $\delta^{15} \mathrm{~N}$ isotopic signal of the basal sources (the macroalgae). The $\delta^{15} \mathrm{~N}$ signatures of some consumers did not follow the marginal vs. central pattern, which indicates that other factors, apart from canopy loss, are feasibly affecting trophic linkages.

We also detected evident temporal patterns in stable nitrogen and carbon isotopes. Several consumers and the algae Corallina spp. were ${ }^{15} \mathrm{~N}$-enriched and ${ }^{13} \mathrm{C}$-depleted in July compared to October, at both marginal and central locations. Temporal shifts in the diets of consumers, as well as changes in the metabolic routes during the growth cycle of organisms and the use of different $\mathrm{N}$ sources by primary producers, may be involved in these variations. For instance, the proximity of the isotopic signal of Rissoa spp. to the signatures of Corallina spp. in October, but not in July, suggested seasonal changes in the gastropod diet (Norderhaug et al. 2003 and references therein), with the inclusion of these algae (as previously reported by Borja 1986) only in October. On the other hand, temporal differences in isotopic ratios of macroalgae could be caused by changes in the biochemical processes during their growth cycle (Fredriksen 2003) and by the use of different $N$ sources by primary producers throughout the year (Viana \& Bode 2013). Also, the great isotopic differentiation between vegetative and reproductive parts of the thalli of $F$. serratus could be linked to withinthallus variation in biochemical processes (Brenchley et al. 1997). Intra-thallus variation in isotopic ratios has been previously reported by Fredriksen (2003) between new and old parts of the kelp Laminaria hyperborea.

Species loss at the current pace is often a consequence of recent climate change and other anthropogenic stressors (Harley et al. 2006, Strain et al. 2014). Furthermore, when key species are also the most prone to become extinct, their loss could trigger major changes in the whole system, spreading further up the food web. This is the case of canopyforming algae, which are among the first group lost in degraded coastal systems (Eriksson et al. 2006), and are very sensitive to climate change (Jueterbock et al. 2013). Indeed, recent studies have reported the contraction of the southern boundary for several fucoids and kelps in the Iberian Peninsula as shifts linked to recent ocean warming (e.g. Fernández 2011, Duarte et al. 2013, Nicastro et al. 2013). These species of fucoids and kelps usually do not have functional equivalents in marine habitats (Schiel 2006). As more productive systems tend to support longer food chains and tangled food webs (Thompson et al. 2007 and references therein), canopy losses would 
lead to the decline of diversity and food-web complexity. Our results are in accordance with the scarce empirical analyses performed to date, detecting this sort of response following canopy loss (Graham 2004, Byrnes et al. 2011). The benthic invertebrate assemblage and their trophic linkages shifted following the disappearance of the canopy-forming alga F. serratus at its retreating range edge. More importantly, the loss of F. serratus may imply a decrease in food chain length and food-web complexity, which feasibly affects higher trophic levels, as fishes and seabirds feed on benthic fauna (Fredriksen 2003 and references therein). Under present and future climatic scenarios, the shifts in the distribution of ecosystem engineers could then entail a reorganization of local natural assemblages and coastal food webs. More attention should be given to measure how much these shifts can modify whole coastal food webs and their functioning.

Acknowledgements. We are grateful to Melissa Foner for her useful comments on the English and Brezo Martínez for helping in the collection of samples. We thank 3 anonymous referees, whose valuable comments greatly helped us to improve our paper in content and presentation. Funding was provided by the Spanish Ministry of Science and Innovation (project GCL2007-66095, contract to L.D. with CGL2010-1931).

\section{LITERATURE CITED}

Airoldi L, Balata D, Beck MW (2008) The Gray Zone: relationships between habitat loss and marine diversity and their applications in conservation. J Exp Mar Biol Ecol 366:8-15

Anderson MJ, Gorley RN, Clarke KR (2008) PERMANOVA for PRIMER: guide to software and statistical methods. PRIMER-E, Plymouth

Arrontes J (1990) Diet, food preference and digestive efficiency in intertidal isopods inhabiting macroalgae. J Exp Mar Biol Ecol 139:231-249

- Arrontes J (1993) Nature of the distributional boundary of Fucus serratus on the north shore of Spain. Mar Ecol Prog Ser 93:183-193

Arrontes J (1999) On the evolution of interactions between marine meso-herbivores and algae. Bot Mar 42:137-155

> Arrontes J (2002) Mechanisms of range expansion in the intertidal brown alga Fucus serratus in Northern Spain. Mar Biol 141:1059-1067

- Baeta A, Valiela I, Rossi F, Pinto R, Richard P, Niquil N, Marques JC (2009) Eutrophication and trophic structure in response to the presence of the eelgrass Zostera noltii. Mar Biol 156:2107-2120

Barry MD (1981) Distribution and ecology of oysters, Ostrea edulis (L.) in Kilkieran and Bertraghboy Bays, Connemara, Co. Galway. Ir Fish Invest B 24:1-12

> Bates CR, DeWreede RE (2007) Do changes in seaweed biodiversity influence associated invertebrate epifauna? J Exp Mar Biol Ecol 344:206-214
Best RJ, Chaudoin AL, Bracken MES, Graham MH, Stachowicz JJ (2014) Plant-animal diversity relationships in a rocky intertidal system depend on invertebrate body size and algal cover. Ecology 95:1308-1322

Bhatnagar MC, Bansal G (2009) Non-Chordata (invertebrate-zoology). Krishna Prakashan Media P, Meerut

$>$ Bode A (1989) Production of the intertidal chiton Acanthochitona crinita within a community of Corallina elongata (Rhodophyta). J Molluscan Stud 55:37-44

> Bode A, Alvarez-Ossorio MT, Varela M (2006) Phytoplankton and macrophyte contributions to littoral food webs in the Galician upwelling estimated from stable isotopes. Mar Ecol Prog Ser 318:89-102

Borja A (1986) La alimentación y distribución del espacio en tres moluscos gasterópodos: Rissoa parva (Da Costa), Barleeia unifasciata (Montagu), y Bittium reticulatum (Da Costa). Cah Biol Mar 29:69-75

Bracken MES, Friberg SE, Gonzalez-Dorantes CA, Williams SL (2008) Functional consequences of realistic biodiversity changes in a marine ecosystem. Proc Natl Acad Sci USA 105:924-928

Brenchley JL, Raven JA, Johnston AM (1997) Resource acquisition in two intertidal fucoid seaweeds, Fucus serratus and Himanthalia elongata: seasonal variation and effects of reproductive development. Mar Biol 129: 367-375

Buschmann AH (1990) Intertidal macroalgae as refuge and food for amphipoda in Central Chile. Aquat Bot 36: $237-245$

> Bustamante RH, Branch GM (1996) The dependence of intertidal consumers on kelp-derived organic matter on the west coast of South Africa. J Exp Mar Biol Ecol 196: $1-28$

Byrnes JE, Reed DC, Cardinale BJ, Cavanaugh KC, Holbrook SJ, Schmitt RJ (2011) Climate-driven increases in storm frequency simplify kelp forest food webs. Glob Change Biol 17:2513-2524

> Camus PA, Daroch K, Opazo LF (2008) Potential for omnivory and apparent intraguild predation in rocky intertidal herbivore assemblages from northern Chile. Mar Ecol Prog Ser 361:35-45

> Cannicci M, Gomei M, Boddi B, Vannini M (2002) Feeding habits and natural diet of the intertidal crab Pachygrapsus marmoratus: opportunistic browser or selective feeder? Estuar Coast Shelf Sci 54:983-1001

> Caut S, Angulo E, Courchamp F (2009) Variation in discrimination factors $\left(\Delta^{15} \mathrm{~N}\right.$ and $\left.\Delta^{13} \mathrm{C}\right)$ : the effect of diet isotopic values and applications for diet reconstruction. J Appl Ecol 46:443-453

Chevreux É, Fage L (1925) Faune de France, Amphipodes. Féderation Francaise des Sociétés de Sciences Naturelles, Paris

> Christie H, Fredriksen S, Rinde E (1998) Regrowth of kelp and colonization of epiphyte and fauna community after kelp trawling at the coast of Norway. Hydrobiologia 375/376:49-58

Christie H, Norderhaug KM, Fredriksen S (2009) Macrophytes as habitat for fauna. Mar Ecol Prog Ser 396: 221-233

Clarke KR (1993) Non-parametric multivariate analyses of changes in community structure. Aust J Ecol 18:117-143

Coll M, Schmidt A, Romanuk T, Lotze HK (2011) Food-web structure of seagrass communities across different spatial scales and human impacts. PLoS ONE 6:e22591, doi: 10.1371/journal.pone.0022591 
Crothers JH (2001) Common topshells: an introduction to the biology of Osilinus lineatus with notes on other species in the genus. Field Stud 10:115-160

> Dayton PK (1985) Ecology of kelp communities. Annu Rev Ecol Syst 16:215-245

DeNiro MJ, Epstein S (1978) Influence of diet on the distribution of carbon isotopes in animals. Geochim Cosmochim Acta 42:495-506

> Duarte L, Viejo RM, Martínez B, deCastro M, GómezGesteira M, Gallardo T (2013) Recent and historical range shifts of two canopy-forming seaweeds in North Spain and the link with trends in sea surface temperature. Acta Oecol 51:1-10

$>$ Duffy JE, Hay ME (1991) Amphipods are not all created equal: a reply to Bell. Ecology 72:354-358

Eriksson BK, Rubach A, Hillebrand H (2006) Biotic habitat complexity controls species diversity and nutrient effects on net biomass production. Ecology 87:246-254

Eriksson BK, Rubach A, Hillebrand H (2007) Dominance by a canopy forming seaweed modifies resource and consumer control of bloom-forming macroalgae. Oikos 116: 1211-1219

> Fernández C (2011) The retreat of large brown seaweeds on the north coast of Spain: the case of Saccorhiza polyschides. Eur J Phycol 46:352-360

Fischer-Piette E (1957) Sur des deplacements de frontières biogeographiques, observés au long des côtes ibèriques dans le domaine intercotidal. Publ Inst Biol Apl 26:35-40

Forero MG, Hobson KA (2003) Using stable isotopes of nitrogen and carbon to study seabird ecology: applications in the Mediterranean seabird community. Sci Mar 67:23-32

Fredriksen S (2003) Food web studies in a Norwegian kelp forest based on stable isotope $\left(\delta^{13} \mathrm{C}\right.$ and $\left.\delta^{15} \mathrm{~N}\right)$ analysis. Mar Ecol Prog Ser 260:71-81

Fretter V, Manly R (1977) Algal associations of Tricolia pullus, Lacuna vinata and Ceriothiopsis tubercularis (Gastropoda) with special reference to the settlement of their larvae. J Mar Biol Assoc UK 57:999-1017

González E, Méndez MG (1986) Crustáceos decápodos das Costas de Galicia. I. Brachyura. Cuadernos de Área de Ciencias Biolóxicas, Seminario de Estudios Gallegos, Ediciós do Castro, A Coruña

> Graham MH (2004) Effects of local deforestation on the diversity and structure of southern California giant kelp forest food webs. Ecosystems 7:341-357

> Guerra-García JM, de Figueroa T, Navarro-Barranco C, Ros M, Sánchez-Moyano JE, Moreira J (2014) Dietary analysis of the marine Amphipoda (Crustacea: Peracarida) from the Iberian Peninsula. J Sea Res 85:508-517

Guiry MD, Guiry GM (2014) AlgaeBase. www.algaebase. org

> Hacker SD, Steneck RS (1990) Habitat architecture and the abundance and body-size-dependent habitat selection of a phytal amphipod. Ecology 71:2269-2285

> Harley CDG, Randall Hughes A, Hultgren KM, Miner BG and others (2006) The impacts of climate change in coastal marine systems. Ecol Lett 9:228-241

> Hawkins SJ, Sugden HE, Mieszkowska N, Moore PJ and others (2009) Consequences of climate-driven biodiversity changes for ecosystem functioning of North European rocky shores. Mar Ecol Prog Ser 396:245-259

- Henriques-Oliveira AL, Nessimian JL, Dorville LFM (2003) Habits of chironomid larvae (Insecta: Diptera) from a stream in the Floresta da Tijuca, Rio de Janeiro, Brazil. Braz J Biol 63:269-281
Hill JM, McQuaid CD (2008) $\delta^{13} \mathrm{C}$ and $\delta^{15} \mathrm{~N}$ biogeographic trends in rocky intertidal communities along the coast of South Africa: evidence of strong environmental signatures. Estuar Coast Shelf Sci 80:261-268

- Holdich DM (1976) A comparison of the ecology and life cycles of two species of littoral isopod. J Exp Mar Biol Ecol 24:133-149

> Hooper DU, Chapin FS III, Ewel JJ, Hector A and others (2005) Effects of biodiversity on ecosystem functioning: a consensus of current knowledge. Ecol Monogr 75:3-35

Jones CG, Lawton JH, Shachak M (1997) Positive and negative effects of organisms as physical ecosystem engineers. Ecology 78:1946-1957

Jueterbock A, Tyberghein L, Verbruggen H, Coyer JA, Olsen JL, Hoarau G (2013) Climate change impact on seaweed meadow distribution in the North Atlantic rocky intertidal. Ecol Evol 3:1356-1373

> Lachnit T, Fischer M, Künzel S, Baines JF, Harder T (2013) Compounds associated with algal surfaces mediate epiphytic colonization of the marine macroalga Fucus vesiculosus. FEMS Microb Ecol 84:411-420

- Laurand S, Riera P (2006) Trophic ecology of the supralittoral rocky shore (Roscoff, France): a dual stable isotope $\left(\delta^{13} \mathrm{C}\right.$, $\left.\delta^{15} \mathrm{~N}\right)$ and experimental approach. J Sea Res 56:27-36

Legendre P, Legendre L (1998) Numerical ecology. Elsevier, Amsterdam

Lilley SA, Schiel DR (2006) Community effects following the deletion of a habitat-forming alga from rocky marine shores. Oecologia 148:672-681

Lima FP, Ribeiro PA, Queiroz N, Hawkins SJ, Santos AM (2007) Do distributional shifts of northern and southern species of algae match the warming pattern? Glob Change Biol 13:2592-2604

Lincoln RJ (1979) British marine Amphipoda: Gammaridea. Pisces Conservation Ltd, British Museum (Natural History), London

Llera EM, Ortea JA, Vizcaino A (1983) Fauna marina de Asturias I. Consejería de Agricultura y Pesca del Principado de Asturias, Asturias

Lüning K (1990) Seaweeds: their environment, biogeography and ecophysiology. Wiley-Interscience, New York, NY

> Mancinelli G, Sabetta L, Basset A (2005) Short-term patch dynamics of macroinvertebrate colonization on decaying reed detritus in a Mediterranean lagoon (Lake Alimini Grande, Apulia, SE Italy). Mar Biol 148:271-283

Martínez B, Viejo RM, Carreño F, Aranda SC (2012a) Habitat distribution models for intertidal seaweeds: responses to climate and to non-climatic drivers. J Biogeogr 39: 1877-1890

> Martínez B, Arenas F, Rubal M, Burgués S and others (2012b) Physical factors driving intertidal macroalgae distribution: physiological stress of a dominant fucoid at its southern limit. Oecologia 170:341-353

- Mateo MA, Serrano O, Serrano L, Michener RH (2008) Effects of sample preparation on stable isotope ratios of carbon and nitrogen in marine invertebrates: implications for food web studies using stable isotopes. Oecologia 157:105-115

> McCutchan JH, Lewis WM, Kendall C, McGrath CC (2003) Variation in trophic shift for stable isotope ratios of carbon, nitrogen, and sulfur. Oikos 102:378-390

Michel L (2011) Multidisciplinary study of trophic diversity and functional role of amphipod crustaceans associated to Posidonia oceanica meadows. PhD dissertation, Uni- 
versity of Liège

Morán JAG, Arrontes J (1994) Factors affecting food preference in a widespread intertidal isopod. J Exp Mar Biol Ecol 182:111-121

$>$ Neues F, Ziegler A, Epple M (2007) The composition of the mineralized cuticle in marine and terrestrial isopods: a comparative study. CrystEngComm 9:1245-1251

Nicastro KR, Zardi GI, Teixeira S, Neiva J, Serrão EA, Pearson GA (2013) Shift happens: trailing edge contraction associated with recent warming trends threatens a distinct genetic lineage in the marine macroalga Fucus vesiculosus. BMC Biol 11:1-13

- Norderhaug KM, Fredriksen S, Nygaard K (2003) Trophic importance of Laminaria hyperborea to kelp forest consumers and the importance of bacterial degradation to food quality. Mar Ecol Prog Ser 255:135-144

Parnell AC, Inger R, Bearhop S, Jackson AL (2010) Source partitioning using stable isotopes: coping with too much variation. PLoS ONE 5:e9672, doi:10.1371/journal.pone. 0009672

Pavia H, Toth G, Åberg P (1999) Trade-offs between phlorotannin production and annual growth in natural populations of the brown seaweed Ascophyllum nodosum. J Ecol 87:761-771

> Peterson BJ, Fry B (1987) Stable isotopes in ecosystem studies. Annu Rev Ecol Syst 18:293-320

Poore AGB (1994) Selective herbivory by amphipods inhabiting the brown alga Zonaria angustata. Mar Ecol Prog Ser 107:113-123

Post DM (2002) Using stable isotopes to estimate trophic position: models, methods, and assumptions. Ecology 83: 703-718

Purchon RD (1977) The biology of the Mollusca, 2nd edn. Pergamon Press, Oxford

Rau GH, Mearns AJ, Young DR, Olson RJ, Schafer HA, Kaplan IR (1983) Animal ${ }^{13} \mathrm{C} /{ }^{12} \mathrm{C}$ correlates with trophic level in pelagic food webs. Ecology 64:1314-1318

R-Core Team (2012). R: A language and environment for statistical computing. R Foundation for Statistical Computing, Vienna. www.R-project.org/

Riera P, Escaravage C, Leroux C (2009) Trophic ecology of the rocky shore community associated with the Ascophyllum nodosum zone (Roscoff, France): a $\delta^{13} \mathrm{C}$ vs $\delta^{15} \mathrm{~N}$ investigation. Estuar Coast Shelf Sci 81:143-148

Roca-Torecilla I, Guerra-García JM (2012) Feeding habits of the peracarid crustaceans associated to the alga Fucus spiralis in Tarifa Island, Cádiz (Southern Spain). Zool Baetica 3:39-47

Rossi F, Baeta A, Marques JC (2015) Stable isotopes reveal habitat-related diet shifts in facultative deposit-feeders. J Sea Res 95:172-179

Salemaa H (1987) Herbivory and microhabitat preferences of Idotea spp. (Isopoda) in the northern Baltic Sea. Ophelia 27:1-15

Schaal G, Riera P, Leroux C, Grall J (2010) A seasonal stable isotope survey of the food web associated to a peri-urban rocky shore. Mar Biol 157:283-294

Schaal G, Riera P, Leroux C (2011) Microscale variation of food web functioning within a rocky shore invertebrate community. Mar Biol 158:623-630

Editorial responsibility: Antonio Bode, A Coruña, Spain
Schiel DR (2006) Rivets or bolts? When single species count in the function of temperate rocky reef communities. J Exp Mar Biol Ecol 338:233-252

> Stephenson RL, Tan FC, Mann KH (1986) Use of stable carbon isotope ratios to compare plant material and potential consumers in a seagrass bed and a kelp bed in Nova Scotia, Canada. Mar Ecol Prog Ser 30:1-7

Strain EMA, Thomson RJ, Micheli F, Mancuso FP, Airoldi L (2014) Identifying the interacting roles of stressors in driving the global loss of canopy-forming to mat-forming algae in marine ecosystems. Glob Change Biol 20: 3300-3312

Tallmark B (1980) Population dynamics of Nassarius reticulatus (Gastropoda, Prosobranchia) in Gullmar Fjord, Sweden. Mar Ecol Prog Ser 3:51-62

Taylor RB, Steinberg PD (2005) Host use by Australasian seaweed mesograzers in relation to feeding preferences of larger grazers. Ecology 86:2955-2967

Thompson RM, Hemberg M, Starzomski BM, Shurin JB (2007) Trophic levels and trophic tangles: the prevalence of omnivory in real food webs. Ecology 88:612-617

Underwood AJ (1997) Experiments in ecology: their logical design and interpretation using analysis of variance. Cambridge University Press, Cambridge

> Van Alstyne KL, Ehlig JM, Whitman SL (1999) Feeding preferences for juvenile and adult algae depends on algal stage and herbivore species. Mar Ecol Prog Ser 180: 179-185

Vander Zanden MJ, Rasmussen JB (2001) Variation in $\delta^{15} \mathrm{~N}$ and $\delta^{13} \mathrm{C}$ trophic fractionation: implications for aquatic food web studies. Limnol Oceanogr 46:2061-2066

> Vanderklift MA, Ponsard S (2003) Sources of variation in consumer-diet $\delta^{15} \mathrm{~N}$ enrichment: a meta-analysis. Oecologia 136:169-182

> Viana IG, Bode A (2013) Stable nitrogen isotopes in coastal macroalgae: geographic and anthropogenic variability. Sci Total Environ 443:887-895

Viejo RM, Arrontes J (1992) Interactions between mesograzers inhabiting Fucus vesiculosus in northern Spain. J Exp Mar Biol Ecol 162:97-111

- Viejo RM, Martínez B, Arrontes J, Astudillo C, Hernández L (2011) Reproductive patterns in central and marginal populations of a large brown seaweed: drastic changes at the southern range limit. Ecography 34:75-84

> Wernberg T, Russell BD, Thomsen MS, Gurgel FD, Bradshaw CJA, Poloczanska ES, Connell SD (2011) Seaweed communities in retreat from ocean warming. Curr Biol 21:1828-1832

Wikström SA, Kautsky L (2007) Structure and diversity of invertebrate communities in the presence and absence of canopy-forming Fucus vesiculosus in the Baltic Sea. Estuar Coast Shelf Sci 72:168-176

> Williams MJ (1981) Methods for analysis of natural diet in portunid crabs (Crustacea: Decapoda: Portunidae). J Exp Mar Biol Ecol 52:103-113

Withers RG, Farnham WF, Lewey S, Jephson NA, Haythorn JM, Gray PWG (1975) The epibionts of Sargassum muticum in British waters. Mar Biol 31:79-86

WoRMS Editorial Board (2014) World Register of Marine Species. www.marinespecies.org (accessed Feb 2014)

Submitted: October 9, 2014; Accepted: February 18, 2015 Proofs received from author(s): April 24, 2015 